\title{
On implicit impulsive Langevin equation involving mixed order derivatives
}

\author{
Akbar Zada', Rizwan Rizwan' ${ }^{1}$ Jiafa Xu² and Zhengqing Fu*
}

\section{"Correspondence:} fzhqing@163.com

${ }^{3}$ College of Mathematics and System Sciences, Shandong University of Science and Technology, Qingdao, China Full list of author information is available at the end of the article

\section{Springer}

\begin{abstract}
In this paper, we consider a nonlocal boundary value problem of nonlinear implicit impulsive Langevin equation involving mixed order derivatives. Sufficient conditions are constructed to discuss the qualitative properties like existence and Ulam's stability of the proposed problem. The main result is verified by an example.

MSC: 26A33; 34A08; 34B27

Keywords: Langevin equation; Caputo derivative; Non-instantaneous impulses; Ulam-Hyers-Rassias stability
\end{abstract}

\section{Introduction}

An equation of the form $m \frac{d^{2} z}{d w^{2}}=\lambda \frac{d z}{d w}+\eta(w)$ is called Langevin equation, introduced by Paul Langevin in 1908. Langevin equations have been broadly used to describe stochastic problems in image processing, physics, astronomy, chemistry, defence system, electrical and mechanical engineering. Brownian motion is well described by the Langevin equations when the random oscillation force is supposed to be Gaussian noise. For the removal of noise, mathematicians used fractional order differential equations, also they perform well in reducing the staircase effects as compared to ordinary differential equations. Thus it is very important to learn the idea of fractional Langevin equations; for more details, see $[1-4]$.

Fractional differential equations (FDEs) provide an excellent tool for the description of memory and hereditary properties of different processes and materials. So, contrary to the classical derivative, the fractional derivative is nonlocal. Fractional calculus has played an important role in enhancing the mathematical modeling of several phenomena appearing in engineering and scientific disciplines, such as blood flow systems, control theory, aerodynamics, the nonlinear oscillation of earthquake, polymer rheology, regular variation in thermodynamics, etc. It has been observed that FDEs are more accurate than the integer-order derivatives. Therefore in the last decades, fractional calculus got considerable attention from researchers, see [5-52].

It is well known that the effects of a pulse are inevitable in many processes and phenomena. For example, in the population dynamics systems, there are abrupt changes in population size due to the effects such as diseases, harvesting, and so on. So, researchers have used impulsive differential equations to describe the aforesaid kinds of phenomena.

(c) The Author(s) 2019. This article is distributed under the terms of the Creative Commons Attribution 4.0 International License (http://creativecommons.org/licenses/by/4.0/), which permits unrestricted use, distribution, and reproduction in any medium, provided you give appropriate credit to the original author(s) and the source, provide a link to the Creative Commons license, and indicate if changes were made. 
In recent times, impulsive fractional differential equations are well investigated with different approaches, we recommend the reader to [53-61].

In fields such as numerical analysis, optimization theory, and nonlinear analysis, mostly we deal with the approximate solutions, and hence we need to check how close these solutions are to the actual solutions of the related system or systems. Many approaches can be used for this purpose, but the Ulam-Hyers stability approach is a simple and easy one. The aforesaid stability was first pointed out by Ulam in 1940 [62] and then solved brilliantly by Hyers in 1941 [63]. Afterwards, stability of such form has been known as Ulam-Hyers stability. In 1978, Rassias [64] generalized the Ulam-Hyers approach by considering variables. Thereafter, mathematicians extended the notions for functional, differential, integrals as well as FDEs [65-72].

Recently, many mathematicians have devoted considerable attention to the existence, uniqueness, and different types of Hyers-Ulam stability of the solutions of nonlinear implicit fractional differential equations with Caputo fractional derivative, see [73-75].

Wang et al. in [76] studied generalized Ulam-Hyers-Rassias stability of the following fractional differential equation:

$$
\left\{\begin{array}{l}
{ }^{c} \mathcal{D}_{0, w}^{v} z(w)=f(w, z(w)), \quad w \in\left(w_{k}, s_{k}\right], k=0,1, \ldots, m, 0<v<1 \\
z(w)=g_{k}(w, z(w)), \quad w \in\left(s_{k-1}, w_{k}\right], k=1,2, \ldots, m
\end{array}\right.
$$

Zada et al. [77] studied the existence and uniqueness of solutions by using Diaz Margolis's fixed point theorem and presented different types of Ulam-Hyers stability for a class of nonlinear implicit fractional differential equations with non-instantaneous integral impulses and nonlinear integral boundary conditions:

$$
\left\{\begin{array}{l}
{ }^{c} \mathcal{D}_{0, w}^{v} z(w)=f\left(w, z(w),{ }^{c} \mathcal{D}_{0, w}^{v} z(w)\right), \quad w \in\left(w_{k}, s_{k}\right], k=0,1, \ldots, m, 0<v<1, w \in(0,1] \\
z(w)=I_{s_{k-1}, w_{k}}^{v}\left(\xi_{k}(w, z(w))\right), \quad w \in\left(s_{k-1}, w_{k}\right], k=0,1, \ldots, m, \\
z(0)=\frac{1}{\Gamma v} \int_{0}^{T}(T-s)^{v-1} \eta(s, z(s)) d s .
\end{array}\right.
$$

Zada et al. [78] studied the existence and uniqueness of solutions by using Diaz Margolis's fixed point theorem and presented different types of Ulam-Hyers stability for a class of nonlinear implicit sequential fractional differential equations with non-instantaneous integral impulses and multi-point boundary conditions:

$$
\left\{\begin{array}{l}
{ }^{c} \mathcal{D}_{0, w}^{v}(\mathcal{D}+\lambda) z(w)=f\left(w, z(w),{ }^{c} \mathcal{D}_{0, w}^{v}\right), \quad w \in\left(w_{k}, s_{k}\right], k=0,1, \ldots, m, 0<v \leq 1 \\
z(w)=g_{k}(w, z(w)), \quad w \in\left(s_{k-1}, w_{k}\right], k=1,2, \ldots, m, \\
z(0)=0, \quad z\left(w_{k}\right)=0, \quad k=0,1, \ldots, m .
\end{array}\right.
$$

In this paper, we study the following nonlocal boundary value problem of nonlinear implicit impulsive Langevin equation with mixed derivatives:

$$
\left\{\begin{array}{l}
{ }^{c} \mathcal{D}_{0, w}^{v}(\mathcal{D}+\lambda) z(w)=f\left(w, z(w),{ }^{c} \mathcal{D}_{0, w}^{v}(\mathcal{D}+\lambda) z(w)\right), \\
\quad w \in\left(w_{k}, s_{k}\right], k=0,1, \ldots, m, \\
z(w)=g_{k}(w, z(w)), \quad w \in\left(s_{k-1}, w_{k}\right], k=1,2, \ldots, m, \\
z(0)=z_{0}, \quad z(T)=\int_{0}^{\eta} \frac{1}{\Gamma p}(\eta-s)^{p-1} z(s) d s, \quad 0<\eta<T,
\end{array}\right.
$$


where ${ }^{c} \mathcal{D}_{0, w}^{v}$ represents the classical Caputo derivative [8] of order $v$ with the lower bound zero, $0=w_{0}<s_{0}<w_{1}<s_{1}<\cdots<w_{m}<s_{m}=\tau, \tau$ is the pre-fixed number, and $\lambda \in \mathbb{R} \backslash\{0\}$, $0<\nu<1, p>0, z_{0}$ are constants, $f:[0, \tau] \times \mathbb{R} \times \mathbb{R} \rightarrow \mathbb{R}$ is continuous and $g_{k}:\left[s_{k-1}, w_{k}\right] \times$ $\mathbb{R} \rightarrow \mathbb{R}$ is continuous for all $k=1,2, \ldots, m$.

In the second section of this paper, we create a uniform framework to originate appropriate formula of solutions for our proposed model. In Sect. 3, we implement the concept of generalized Ulam-Hyers-Rassias stability of Eq. (1.2). Finally, we give an example which supports our main result.

\section{Preliminaries}

Let $J=[0, \tau]$ and $C(J, \mathbb{R})$ be the space of all continuous functions from $J$ to $\mathbb{R}$ and a piecewise continuous functions space $P C(J, \mathbb{R})=\left\{z: f \rightarrow \mathbb{R}: z \in\left(\left(w_{k}, w_{k-1}\right)\right], \mathbb{R}\right), k=$ $0,1, \ldots, m$, and there exist $z\left(w_{k}^{-}\right)$and $z\left(w_{k}^{+}\right), k=1,2, \ldots, m$, with $\left.z\left(w_{k}^{-}\right)=z\left(w_{k}^{+}\right)\right\}$.

Consider the linear form of (1.1) as follows:

$$
\left\{\begin{array}{l}
{ }^{c} \mathcal{D}_{0, t}^{v}(\mathcal{D}+\lambda) z(w)=f(w), \quad w \in\left(w_{k}, s_{k}\right], k=0,1, \ldots, m, 0<v<1, \\
z(w)=g_{k}(w), \quad w \in\left(s_{k-1}, w_{k}\right], k=1,2, \ldots, m, \\
z(0)=z_{0}, \quad z(T)=\theta I^{p} z(\eta) \\
\quad \text { where } I^{p} z(\eta)=\int_{0}^{\eta} \frac{1}{\Gamma p}(\eta-s)^{p-1} z(s) d s, 0<\eta<T .
\end{array}\right.
$$

We recall some definitions of fractional calculus from [5] as follows.

Definition 2.1 The fractional integral of order $v$ from 0 to $w$ for the function $f$ is defined by

$$
I_{0, w}^{v} f(w)=\frac{1}{\Gamma(v)} \int_{0}^{w} f(s)(w-s)^{v-1} d s, \quad w>0, v>0,
$$

where $\Gamma(\cdot)$ is the gamma function.

Definition 2.2 The Riemann-Liouville fractional derivative of fractional order $v$ from 0 to $w$ for a function $f$ can be written as

$$
{ }^{L} \mathcal{D}_{0, w}^{v} f(w)=\frac{1}{\Gamma(n-v)} \frac{d^{n}}{d t^{n}} \int_{0}^{w} \frac{f(s)}{(w-s)^{v+1-n}} d s, \quad w>0, n-1<v<n,
$$

where $\Gamma(\cdot)$ is the gamma function.

Definition 2.3 The Caputo derivative of fractional order $v$ from 0 to $w$ for a function $f$ can be defined as

$$
{ }^{c} \mathcal{D}_{0, w}^{v} f(w)=\frac{1}{\Gamma(n-v)} \int_{0}^{w}(w-s)^{n-v-1} f^{n}(s) d s, \quad \text { where } n=[v]+1 .
$$

Definition 2.4 The general form of the classical Caputo derivative of order $v$ of a function $f$ can be given as

$$
{ }^{c} \mathcal{D}_{0, w}^{\nu}={ }^{L} \mathcal{D}_{0, w}^{v}\left(f(w)-\sum_{k=0}^{n-1} \frac{w^{k}}{k !} f^{(k)}(0)\right), \quad w>0, n-1<v<n .
$$




\section{Remark 2.5}

(i) If $f(\cdot) \in C^{m}([0, \infty), \mathbb{R})$, then

$$
\begin{aligned}
& { }^{L} \mathcal{D}_{0, w}^{v} f(w)=\frac{1}{\Gamma(m-v)} \int_{0}^{w} \frac{f^{m}(s)}{(w-s)^{v+1-m}} d s=I_{0, w}^{m-v} f^{(m)}(w), \\
& w>0, m-1<v<m
\end{aligned}
$$

(ii) In Definition 2.4, the integrable function $f$ can be discontinuous function. This fact can support us in considering impulsive fractional problems in the sequel.

Lemma 2.6 ([5]) The fractional differential equation ${ }^{c} D^{v} f(w)=0$ with $v>0$, involving Caputo differential operator ${ }^{c} D^{v}$, has a solution in the following form:

$$
f(w)=c_{0}+c_{1} w+c_{2} w^{2}+\cdots+c_{m-1} w^{n-1},
$$

where $c_{k} \in \mathbb{R}, k=0,1, \ldots, m-1$, and $m=[v+1]$.

Lemma 2.7 ([5]) For arbitrary $v>0$, we have

$$
I^{v}\left({ }^{c} \mathcal{D}^{v} f(w)\right)=c_{0}+c_{1} w+c_{2} w^{2}+\cdots+c_{m-1} w^{n-1},
$$

where $c_{k} \in \mathbb{R}, k=0,1, \ldots, m-1$, and $m=[v+1]$.

Lemma $2.8([6])$ Let $v>0$ and $\beta>0, f \in L^{1}([a, b])$.

Then

$$
\begin{aligned}
& I^{v} I^{\beta} f(w)=I^{v+\beta} f(w), \quad{ }^{c} \mathcal{D}_{0, w}^{v}\left({ }^{c} \mathcal{D}_{0, w}^{\beta} f(w)\right)={ }^{c} \mathcal{D}_{0, w}^{v+\beta} f(w), \quad \text { and } \\
& I^{v} \mathcal{D}_{0, w}^{v} f(w)=f(w), \quad w \in[a, b] .
\end{aligned}
$$

Lemma 2.9 The function $z \in P C(J, \mathbb{R})$ is a solution of (2.1) if and only if

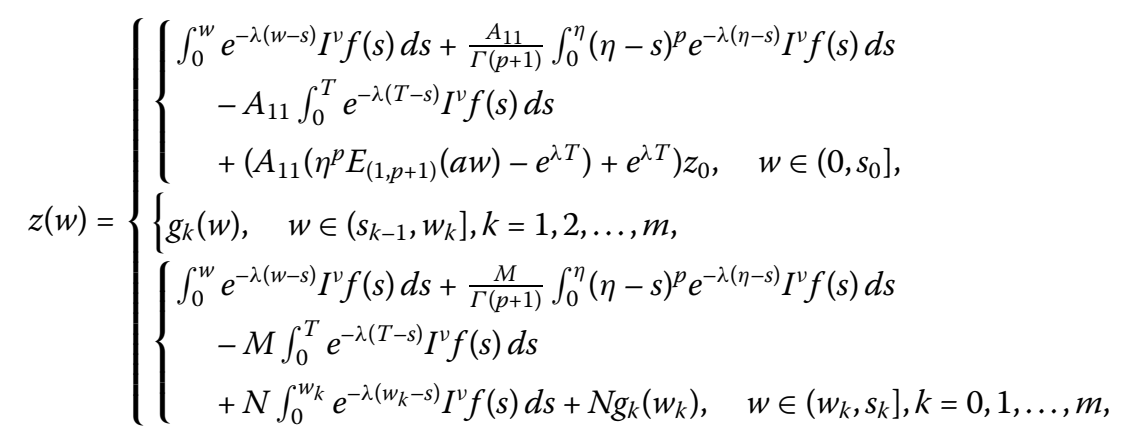

where

$$
\begin{aligned}
& A_{11}=\frac{\lambda \Gamma(p+1)}{\left(1-e^{-\lambda T}\right) \Gamma(p+1)-\eta^{p}+\Gamma(p+1) \eta^{p} E_{(1, p+1)}(a w)}, \\
& B_{k}=\frac{\lambda \Gamma(p+1)\left(\eta^{p} E_{(1, p+1)}(a w)-e^{-\lambda T}\right)}{\delta_{k}}
\end{aligned}
$$




$$
\begin{aligned}
A_{k}= & \frac{\delta_{k} \lambda \Gamma(p+1)-\Gamma(p+1)\left(1-e^{\lambda w_{k}}\right)\left(\lambda \Gamma(p+1)\left(\eta^{p} E_{(1, p+1)}(a w)-e^{-\lambda T}\right)\right)}{\delta_{k}\left(\left(1-e^{-\lambda T}\right) \Gamma(p+1)-\eta^{p}+\Gamma(p+1) \eta^{p} E_{(1, p+1)}(a w)\right)}, \\
M_{k}= & \frac{A_{k}\left(1-e^{-\lambda w}\right)}{\lambda}-\frac{\Gamma(p+1) e^{-\lambda w}\left(1-e^{-\lambda w_{k}}\right)}{\delta_{k}} \\
N_{k}= & \frac{B_{k}\left(1-e^{-\lambda w}\right)}{\lambda}-\frac{\left(1-e^{-\lambda T}\right) \Gamma(p+1)-\eta^{p}+\Gamma(p+1) \eta^{p} E_{(1, p+1)}(a w)}{\delta_{k} e^{\lambda w}} \\
\delta_{k}= & 2 \Gamma(p+1)\left(e^{-\lambda w_{k}}-e^{-\lambda\left(w_{k}+T\right)}+\eta^{p} E_{(1, p+1)}(a w) e^{-\lambda w_{k}}\right) \\
& -\eta^{p} e^{-\lambda w_{k}}-\Gamma(p+1) E_{(1, p+1)}(a w) .
\end{aligned}
$$

Proof Let $z$ be a solution of problem (2.1), we have the following cases.

Case 1: For $w \in\left[0, s_{0}\right]$, we consider

$$
{ }^{c} \mathcal{D}_{0, w}^{v}(\mathcal{D}+\lambda) z(w)=f(w), \quad z(0)=z_{0}, \quad \text { and } \quad z(T)=I^{p} z(\eta),
$$

where $\mathcal{D}$ denotes an ordinary differential operator. In light of Lemma 2.7 and an ordinary integration, we get

$$
z(w)=\int_{0}^{w} e^{-\lambda(w-s)} I^{v} f(s) d s+c_{0}\left(\frac{1-e^{-\lambda w}}{\lambda}\right)+c_{1} e^{-\lambda w} .
$$

Using boundary conditions, we get

$$
\begin{aligned}
z(w)= & \int_{0}^{w} e^{-\lambda(w-s)} I^{v} f(s) d s+\frac{A_{11}}{\Gamma(p+1)} \int_{0}^{\eta}(\eta-s)^{p} e^{-\lambda(\eta-s)} I^{v} f(s) d s \\
& -A_{11} \int_{0}^{T} e^{-\lambda(T-s)} I^{v} f(s) d s+\left(A_{11}\left(\eta^{p} E_{(1, p+1)}(a w)-e^{\lambda T}\right)+e^{\lambda T}\right) z_{0} .
\end{aligned}
$$

For $w \in\left(s_{0}, w_{1}\right], z(w)=g_{1}(w)$.

Case 2: For $w \in\left(w_{1}, s_{1}\right]$, we consider

$$
{ }^{c} \mathcal{D}_{0, w}^{v}(\mathcal{D}+\lambda) z(w)=f(w) \quad \text { with } z\left(w_{1}\right)=g_{1}\left(w_{1}\right) .
$$

Since $z\left(w_{1}\right)=g_{1}\left(w_{1}\right)$, then Eq. (2.2) is of the following type:

$$
g_{1}\left(w_{1}\right)=\int_{0}^{w_{1}} e^{-\lambda\left(w_{1}-s\right)} I^{v} f(s) d s+c_{0}\left(\frac{1-e^{-\lambda w_{1}}}{\lambda}\right)+c_{1} e^{-\lambda w_{1}} .
$$

Using boundary conditions, we get

$$
\begin{aligned}
z(w)= & \int_{0}^{w} e^{-\lambda(w-s)} I^{v} f(s) d s+\frac{M_{1}}{\Gamma(p+1)} \int_{0}^{\eta}(\eta-s)^{p} e^{-\lambda(\eta-s)} I^{v} f(s) d s \\
& -M_{1} \int_{0}^{T} e^{-\lambda(T-s)} I^{v} f(s) d s+N_{1} \int_{0}^{w_{1}} e^{-\lambda\left(w_{1}-s\right)} I^{\nu} f(s) d s+N_{1} g_{1}\left(w_{1}\right),
\end{aligned}
$$

where

$$
A_{11}=\frac{\lambda \Gamma(p+1)}{\left(1-e^{-\lambda T}\right) \Gamma(p+1)-\eta^{p}+\Gamma(p+1) \eta^{p} E_{(1, p+1)}(a w)},
$$




$$
\begin{aligned}
B_{22}= & \frac{\lambda \Gamma(p+1)\left(\eta^{p} E_{(1, p+1)}(a w)-e^{-\lambda T}\right)}{\delta_{1}}, \\
A_{22}= & \frac{\delta_{1} \lambda \Gamma(p+1)-\Gamma(p+1)\left(1-e^{\lambda w_{1}}\right)\left(\lambda \Gamma(p+1)\left(\eta^{p} E_{(1, p+1)}(a w)-e^{-\lambda T}\right)\right)}{\delta_{1}\left(\left(1-e^{-\lambda T}\right) \Gamma(p+1)-\eta^{p}+\Gamma(p+1) \eta^{p} E_{(1, p+1)}(a w)\right)}, \\
M_{1}= & \frac{A_{22}\left(1-e^{-\lambda w}\right)}{\lambda}-\frac{\Gamma(p+1) e^{-\lambda w}\left(1-e^{-\lambda w_{1}}\right)}{\delta_{1}}, \\
N_{1}= & \frac{B_{22}\left(1-e^{-\lambda w}\right)}{\lambda}-\frac{\left(1-e^{-\lambda T}\right) \Gamma(p+1)-\eta^{p}+\Gamma(p+1) \eta^{p} E_{(1, p+1)}(a w)}{\delta_{1} e^{\lambda w}} \\
\delta_{1}= & 2 \Gamma(p+1)\left(e^{-\lambda w_{1}}-e^{-\lambda\left(w_{1}+T\right)}+\eta^{p} E_{(1, p+1)}(a w) e^{-\lambda w_{1}}\right) \\
& -\eta^{p} e^{-\lambda w_{1}}-\Gamma(p+1) E_{(1, p+1)}(a w) .
\end{aligned}
$$

Generally speaking, for $w \in\left(s_{k-1}, w_{k}\right], z\left(w_{k}\right)=g_{k}\left(w_{k}\right)$.

Case 3: For $w \in\left(w_{k}, s_{k}\right]$, we consider

$$
\mathcal{D}_{0, w}^{v}(\mathcal{D}+\lambda) z(w)=f(w) \quad \text { with } z\left(w_{k}\right)=g_{k}\left(w_{k}\right) \quad \text { and } \quad u(T)=I^{p} z(\eta) .
$$

By repeating again the same process, we have

$$
\begin{aligned}
z(w)= & \int_{0}^{w} e^{-\lambda(w-s)} I^{v} f(s) d s+\frac{M_{k}}{\Gamma(p+1)} \int_{0}^{\eta}(\eta-s)^{p} e^{-\lambda(\eta-s)} I^{v} f(s) d s \\
& -M_{k} \int_{0}^{T} e^{-\lambda(T-s)} I^{v} f(s) d s+N_{k} \int_{0}^{w_{k}} e^{-\lambda\left(w_{k}-s\right)} I^{v} f(s) d s+N_{k} g_{k}\left(w_{k}\right),
\end{aligned}
$$

where

$$
\begin{aligned}
A_{11}= & \frac{\lambda \Gamma(p+1)}{\left(1-e^{-\lambda T}\right) \Gamma(p+1)-\eta^{p}+\Gamma(p+1) \eta^{p} E_{(1, p+1)}(a w)}, \\
B_{k}= & \frac{\lambda \Gamma(p+1)\left(\eta^{p} E_{(1, p+1)}(a w)-e^{-\lambda T}\right)}{\delta_{k}} \\
A_{k}= & \frac{\delta_{k} \lambda \Gamma(p+1)-\Gamma(p+1)\left(1-e^{\lambda w_{k}}\right)\left(\lambda \Gamma(p+1)\left(\eta^{p} E_{(1, p+1)}(a w)-e^{-\lambda T}\right)\right)}{\delta_{k}\left(\left(1-e^{-\lambda T}\right) \Gamma(p+1)-\eta^{p}+\Gamma(p+1) \eta^{p} E_{(1, p+1)}(a w)\right)} \\
M_{k}= & \frac{A_{k}\left(1-e^{-\lambda w}\right)}{\lambda}-\frac{\Gamma(p+1) e^{-\lambda w}\left(1-e^{-\lambda w_{k}}\right)}{\delta_{k}} \\
N_{k}= & \frac{B_{k}\left(1-e^{-\lambda w}\right)}{\lambda}-\frac{\left(1-e^{-\lambda T}\right) \Gamma(p+1)-\eta^{p}+\Gamma(p+1) \eta^{p} E_{(1, p+1)}(a w)}{\delta_{k} e^{\lambda w}} \\
\delta_{k}= & 2 \Gamma(p+1)\left(e^{-\lambda w_{k}}-e^{-\lambda\left(w_{k}+T\right)}+\eta^{p} E_{(1, p+1)}(a w) e^{-\lambda w_{k}}\right) \\
& -\eta^{p} e^{-\lambda w_{k}}-\Gamma(p+1) E_{(1, p+1)}(a w) .
\end{aligned}
$$

\section{Stability result}

By the ideas of stability in $[1,65,79]$, we can generate a generalized Ulam-Hyers-Rassias stability concept for Eq. (1.2). 
Let $\epsilon, \psi \geq 0$ and $\varphi \in P C\left(J, \mathbb{R}_{+}\right)$be nondecreasing, consider

$$
\left\{\begin{array}{l}
\left|{ }^{c} \mathcal{D}_{0, w}^{v}(\mathcal{D}+\lambda) z(w)-f\left(w, z(w),{ }^{c} \mathcal{D}_{0, w}^{v}(\mathcal{D}+\lambda) z(w)\right)\right| \leq \varphi(w), \\
\quad w \in\left(w_{k}, s_{k}\right], k=0,1, \ldots, m, 0<v<1, \\
\left|z(w)-N g_{k}(w, z(w))\right| \leq \psi, \quad w \in\left(s_{k-1}, w_{k}\right], k=1,2, \ldots, m
\end{array}\right.
$$

Remark 3.1 A function $z \in P C(J, \mathbb{R})$ is a solution of inequality (3.1) if and only if there are $G \in P C(J, \mathbb{R})$ and a sequence $G_{k}, k=1,2, \ldots, m$ (which depends on $z$ ) such that

(i) $|G(w)| \leq \varphi(w), w \in J$, and $\left|G_{k}\right| \leq \psi, k=1,2, \ldots, m$;

(ii) ${ }^{c} \mathcal{D}_{0, w}^{v}(\mathcal{D}+\lambda) z(w)=f\left(w, z(w),{ }^{c} \mathcal{D}_{0, w}^{v}(\mathcal{D}+\lambda) z(w)\right)+G(w), w \in\left(w_{k}, s_{k}\right], k=1,2, \ldots, m$;

(iii) $z(w)=N_{k} g_{k}(w, z(w))+G_{k}, w \in\left(s_{k-1}, w_{k}\right], k=1,2, \ldots, m$.

Definition 3.2 Equation (1.2) is generalized Ulam-Hyers-Rassias stable with respect to $(\varphi, \psi)$ if there exists $c_{f, v, g_{k}, \varphi}>0$ such that, for each solution $z \in P C(J, \mathbb{R})$ of inequality (3.1), there is a solution $z_{0} \in P C(J, \mathbb{R})$ of Eq. (1.2) with

$$
\left|z(w)-z_{0}(w)\right| \leq c_{f, v, g_{k}, \varphi}(\varphi(w)+\psi), \quad w \in J
$$

Remark 3.3 If $z \in P C(J, \mathbb{R})$ is a solution of inequality (3.1), then $z$ is a solution of the following integral inequality:

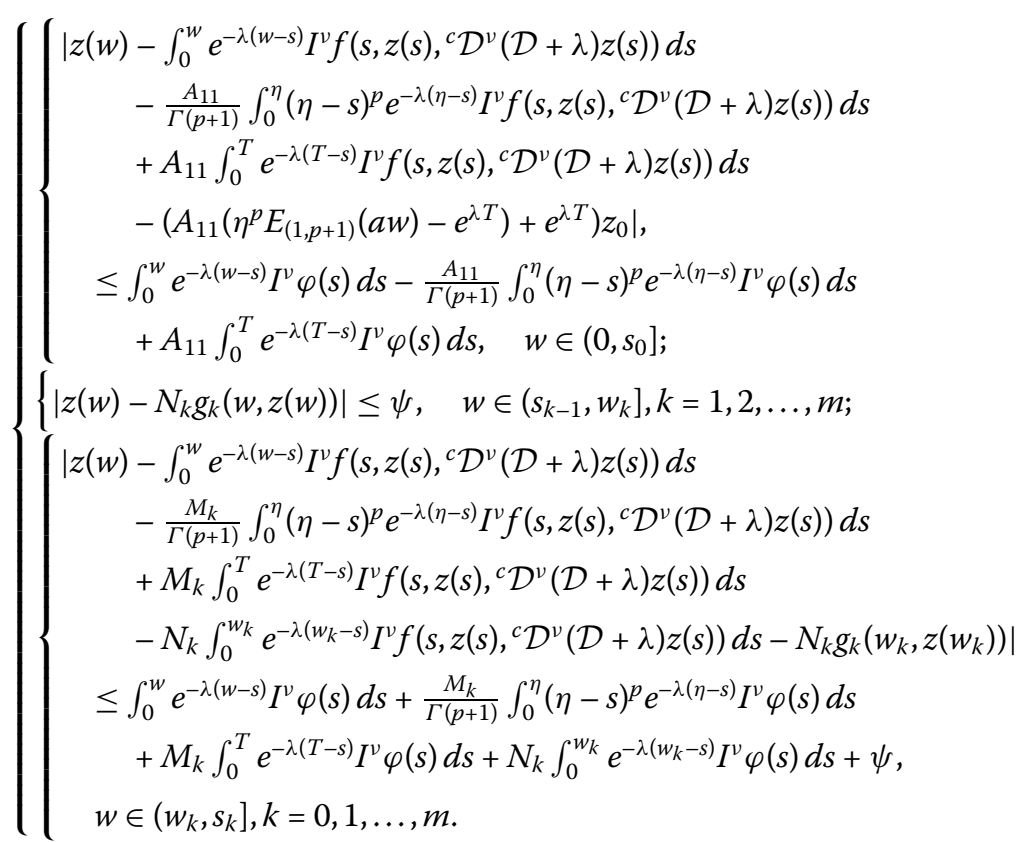

In fact, by Remark 3.1, we get

$$
\left\{\begin{array}{c}
{ }^{c} \mathcal{D}_{0, w}^{v}(\mathcal{D}+\lambda) z(w)=f\left(w, z(w),{ }^{c} \mathcal{D}_{0, w}^{v}(\mathcal{D}+\lambda) z(w)\right)+G(w), \\
\quad w \in\left(w_{k}, s_{k}\right], k=0,1, \ldots, m, 0<v<1, \\
z(w)=N_{k} g_{k}(w, z(w))+G_{k}, \quad w \in\left(s_{k-1}, w_{k}\right], k=1,2, \ldots, m .
\end{array}\right.
$$


Clearly, the solution of Eq. (3.3) is given by

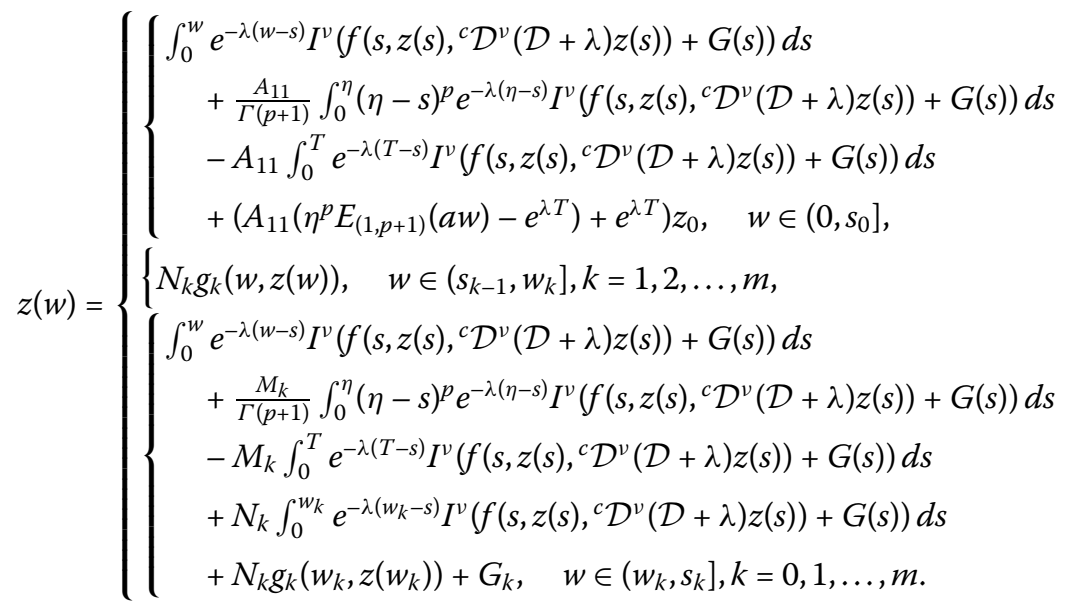

For $w \in\left(w_{k}, s_{k}\right], k=0,1, \ldots, m$, we get

$$
\begin{aligned}
\mid z(w) & -\int_{0}^{w} e^{-\lambda(w-s)} I^{v} f\left(s, z(s),{ }^{c} \mathcal{D}^{v}(\mathcal{D}+\lambda) z(s)\right) d s \\
& -\frac{M_{k}}{\Gamma(p+1)} \int_{0}^{\eta}(\eta-s)^{p} e^{-\lambda(\eta-s)} I^{v} f\left(s, z(s),{ }^{c} \mathcal{D}^{v}(\mathcal{D}+\lambda) z(s)\right) d s-N_{k} g_{k}\left(w_{k}, z\left(w_{k}\right)\right) \\
& +M_{k} \int_{0}^{T} e^{-\lambda(T-s)} I^{v} f\left(s, z(s),{ }^{c} \mathcal{D}^{v}(\mathcal{D}+\lambda) z(s)\right) d s \\
& -N_{k} \int_{0}^{w_{k}} e^{-\lambda\left(w_{k}-s\right)} I^{v} f\left(s, z(s),{ }^{c} \mathcal{D}^{v}(\mathcal{D}+\lambda) z(s)\right) d s \mid \\
\leq & \left|\int_{0}^{w} e^{-\lambda(w-s)} I^{v} G(s) d s\right|+\left|\frac{M_{k}}{\Gamma(p+1)} \int_{0}^{\eta}(\eta-s)^{p} e^{-\lambda(\eta-s)} I^{v} G(s) d s\right| \\
& +\left|M_{k} \int_{0}^{T} e^{-\lambda(T-s)} I^{v} G(s) d s\right|+\left|N_{k} \int_{0}^{w_{k}} e^{-\lambda\left(w_{k}-s\right)} I^{v} G(s) d s\right|+\left|G_{k}\right| \\
\leq & \int_{0}^{w} e^{-\lambda(w-s)} I^{v} \varphi(s) d s+\frac{M_{k}}{\Gamma(p+1)} \int_{0}^{\eta}(\eta-s)^{p} e^{-\lambda(\eta-s)} I^{v} \varphi(s) d s \\
& +M_{k} \int_{0}^{T} e^{-\lambda(T-s)} I^{v} \varphi(s) d s+N_{k} \int_{0}^{w_{k}} e^{-\lambda\left(w_{k}-s\right)} I^{v} \varphi(s) d s+\psi .
\end{aligned}
$$

Proceeding the above, we derive that

$$
\left|z(w)-N_{k} g_{k}(w, z(w))\right| \leq\left|G_{k}\right| \leq \psi, \quad w \in\left(s_{k-1}, w_{k}\right], k=1,2, \ldots, m,
$$

and

$$
\begin{aligned}
\mid z(w) & -\int_{0}^{w} e^{-\lambda(w-s)} I^{v} f\left(s, z(s),{ }^{c} \mathcal{D}^{v}(\mathcal{D}+\lambda) z(s)\right) d s \\
& -\frac{A_{11}}{\Gamma(p+1)} \int_{0}^{\eta}(\eta-s)^{p} e^{-\lambda(\eta-s)} I^{v} f\left(s, z(s),{ }^{c} \mathcal{D}^{v}(\mathcal{D}+\lambda) z(s)\right) d s \\
& +A_{11} \int_{0}^{T} e^{-\lambda(T-s)} I^{v} f\left(s, z(s),{ }^{c} \mathcal{D}^{v}(\mathcal{D}+\lambda) z(s)\right) d s
\end{aligned}
$$




$$
\begin{aligned}
& -\left(A_{11}\left(\eta^{p} E_{(1, p+1)}(a w)-e^{\lambda T}\right)+e^{\lambda T}\right) z_{0} \mid \\
\leq & \left|\int_{0}^{w} e^{-\lambda(w-s)} I^{v} G(s) d s\right|+\left|\frac{A_{11}}{\Gamma(p+1)} \int_{0}^{\eta}(\eta-s)^{p} e^{-\lambda(\eta-s)} I^{v} G(s) d s\right| \\
& +\left|A_{11} \int_{0}^{T} e^{-\lambda(T-s)} I^{v} G(s) d s\right| \\
\leq & \int_{0}^{w} e^{-\lambda(w-s)} I^{v} \varphi(s) d s+\frac{A_{11}}{\Gamma(p+1)} \int_{0}^{\eta}(\eta-s)^{p} e^{-\lambda(\eta-s)} I^{v} \varphi(s) d s \\
& +A_{11} \int_{0}^{T} e^{-\lambda(T-s)} I^{v} \varphi(s) d s, \quad w \in\left(0, s_{0}\right] .
\end{aligned}
$$

\section{Main results}

This section is started with the following definition.

Definition 4.1 For a nonempty set $V$, a function $d: V \times V \rightarrow[0, \infty]$ is called a generalized metric on $V$ if and only if $d$ satisfies:

$\diamond d\left(v_{1}, v_{2}\right)=0$ if and only if $v_{1}=v_{2}$;

$\diamond d\left(v_{1}, v_{2}\right)=d\left(v_{2}, v_{1}\right)$ for all $v_{1}, v_{2} \in V$;

$\diamond d\left(v_{1}, v_{3}\right) \leq d\left(v_{1}, v_{2}\right)+d\left(v_{2}, v_{3}\right)$ for all $v_{1}, v_{2}, v_{3} \in V$.

Lemma 4.2 (see [80] (Generalized Diaz-Margolis's fixed point theorem)) Let $(V, d)$ be a generalized complete metric space. Assume that $T: V \rightarrow V$ is a strictly contractive operator with the Lipschitz constant $L<1$. If there exists $k \geq 0$ such that $d\left(T^{k+1} v, T^{k} v\right)<\infty$ for some $v$ in $V$, then the following statements are true:

$\left(B_{1}\right)$ The sequence $\left\{T^{n} v\right\}$ converges to a fixed point $v^{*}$ of $T$;

$\left(B_{2}\right)$ The unique fixed point of $T$ is $v^{*} \in V^{*}=\left\{u \in V\right.$ such that $\left.d\left(T^{k} v, u\right)<\infty\right\}$;

$\left(B_{3}\right) u \in V^{*}$, then $d\left(u, v^{*}\right) \leq \frac{1}{1-L} d(T u, u)$.

We can introduce some assumptions as follows:

$\left(H_{1}\right) f \in C(J \times \mathbb{R}, \mathbb{R})$.

$\left(H_{2}\right)$ There exists a positive constant $\mathrm{七}_{f}$ such that

$$
\begin{aligned}
& |f(w, u, m)-f(w, v, n)| \leq \mathrm{七}_{f_{1}}|u-v|+\mathrm{七}_{f_{2}}|m-n| \\
& \text { for each } w \in J \text { and all } u, v, m, n \in \mathbb{R} .
\end{aligned}
$$

$\left(H_{3}\right) g_{k} \in C\left(\left(s_{k-1}, w_{k}\right] \times \mathbb{R}, \mathbb{R}\right)$ and there are positive constants $\mathrm{七}_{g k}, k=1,2, \ldots, m$, such that

$$
\left|g_{k}(w, v)-g_{k}(w, v)\right| \leq \mathfrak{七}_{g k}|u-v| \quad \text { for each } w \in\left(s_{k-1}, w_{k}\right] \text { and all } u, v \in \mathbb{R} \text {. }
$$

$\left(H_{4}\right)$ Let $\varphi \in C\left(J, \mathbb{R}_{+}\right)$be a nondecreasing function, there exist $C_{\varphi}, C_{\gamma}>0$ such that

$$
\begin{aligned}
& \int_{0}^{w} I^{\nu}(\varphi(s)) d s \leq C_{\varphi} \varphi(w) \quad \text { for each } w \in J \\
& \int_{0}^{w}(\mathcal{D}+\lambda)(\varphi(s)) d s \leq C_{\gamma} \varphi(w) \quad \text { for each } w \in J .
\end{aligned}
$$


Theorem 4.3 Suppose that $\left(H_{1}\right)-\left(H_{2}\right)$ are satisfied and also a function $z \in P C(J, \mathbb{R})$ satisfies (3.1). Then there exists a unique solution $z_{0}$ of Eq. (1.2) such that

$$
z_{0}(w)=\left\{\begin{array}{l}
\left\{\begin{array}{l}
\int_{0}^{w} e^{-\lambda(w-s)} I^{v} f\left(s, z_{0}(s),{ }^{c} \mathcal{D}^{v}(\mathcal{D}+\lambda) z_{0}(s)\right) d s \\
\quad+\frac{A_{11}}{\Gamma(p+1)} \int_{0}^{\eta}(\eta-s)^{p} e^{-\lambda(\eta-s)} I^{v} f\left(s, z_{0}(s),{ }^{c} \mathcal{D}^{v}(\mathcal{D}+\lambda) z_{0}(s)\right) d s \\
\quad-A_{11} \int_{0}^{T} e^{-\lambda(T-s)} I^{v} f\left(s, z_{0}(s),{ }^{c} \mathcal{D}^{v}(\mathcal{D}+\lambda) z_{0}(s)\right) d s \\
\quad+\left(A_{11}\left(\eta^{p} E_{(1, p+1)}(a w)-e^{\lambda T}\right)+e^{\lambda T}\right) z_{0}, \quad w \in\left(0, s_{0}\right]
\end{array}\right. \\
\left\{\begin{array}{l}
\left\{g_{k}\left(w, z_{0}(w)\right), \quad w \in\left(s_{k-1}, w_{k}\right], k=1,2, \ldots, m,\right. \\
\quad+\frac{M_{k}}{\Gamma(p+1)} \int_{0}^{\eta}(\eta-s){ }^{p} e^{-\lambda(\eta-s)} I^{v} f\left(s, z_{0}(s),{ }^{c} \mathcal{D}^{v}(\mathcal{D}+\lambda) z_{0}(s)\right) d s \\
\quad+M_{k} \int_{0}^{T} e^{-\lambda(T-s)} I^{v} f\left(s, z_{0}(s),{ }^{c} \mathcal{D}^{v}(\mathcal{D}+\lambda) z_{0}(s)\right) d s \\
\quad+N_{k} \int_{0}^{w_{k}} e^{-\lambda\left(w_{k}-s\right)} I^{v} f\left(s, z_{0}(s),{ }^{c} \mathcal{D}^{v}(\mathcal{D}+\lambda) z_{0}(s)\right) d s \\
+N_{k} g_{k}\left(w_{k}, z_{0}\left(w_{k}\right)\right), \quad w \in\left(w_{k}, s_{k}\right]
\end{array}\right.
\end{array}\right.
$$

and

$$
\begin{aligned}
\left|z(w)-z_{0}(w)\right| \leq & \left\{\left(\left(\frac{1-e^{-\lambda w}}{\lambda}\right)+\frac{M_{k}}{\Gamma(p+1)} \frac{\eta^{p+1}}{p+1}\left(\frac{1-e^{-\lambda \eta}}{\lambda}\right)+M_{k}\left(\frac{1-e^{-\lambda T}}{\lambda}\right)\right.\right. \\
& \left.\left.+N_{k}\left(\frac{1-e^{-\lambda w_{k}}}{\lambda}\right)\right)\left(\mathrm{七}_{f_{1}} C_{\varphi}+\mathrm{Ł}_{f_{2}} C_{\gamma}\right)+N_{k} \mathrm{七}_{g k}\right\}\left(\frac{\varphi(w)+\psi}{1-\succeq}\right)
\end{aligned}
$$

for all $w \in J$ if $0<v<1$, and

$$
€=\max \left\{\mathrm{七}_{1}, \mathrm{七}_{2}\right\}<1,
$$

where

$$
\begin{aligned}
\mathrm{Ł}_{1}= & \max \left\{\left(\left(\frac{1-e^{-\lambda w}}{\lambda}\right)+\frac{M_{k}}{\Gamma(p+1)} \frac{\eta^{p+1}}{p+1}\left(\frac{1-e^{-\lambda \eta}}{\lambda}\right)+M_{k}\left(\frac{1-e^{-\lambda T}}{\lambda}\right)\right.\right. \\
& \left.\left.+N_{k}\left(\frac{1-e^{-\lambda w_{k}}}{\lambda}\right)\right)\left(\mathrm{七}_{f_{1}} C_{\varphi}+\mathrm{七}_{f_{2}} C_{\gamma}\right)+N_{k} \mathrm{七}_{g k} \text { such that } k=1,2, \ldots, m\right\}, \\
\mathrm{Ł}_{2}= & \max \left\{\mathrm{七}_{f_{1}}\left(\frac{1-e^{-\lambda w}}{\lambda}\right)\left(\frac{w^{v}}{\Gamma(v+1)}\right)+\mathrm{七}_{f_{2}} \lambda\left(\frac{1-e^{-\lambda w}}{\lambda}\right)\right. \\
& +\mathrm{七}_{f_{2}} \lambda \frac{M_{k}}{\Gamma(p+1)}\left(\frac{\eta^{p+1}}{p+1}\right)\left(\frac{1-e^{-\lambda \eta}}{\lambda}\right) \\
& +\mathrm{七}_{f_{1}} \frac{M_{k}}{\Gamma(p+1)}\left(\frac{\eta^{p+1}}{p+1}\right)\left(\frac{1-e^{-\lambda \eta}}{\lambda}\right)\left(\frac{\eta^{v}}{\Gamma(v+1)}\right)+\mathrm{七}_{f_{1}} M_{k}\left(\frac{1-e^{-\lambda T}}{\lambda}\right)\left(\frac{T^{v}}{\Gamma(v+1)}\right) \\
& +\mathrm{七}_{f_{2}} \lambda M_{k}\left(\frac{1-e^{-\lambda T}}{\lambda}\right)+\mathrm{七}_{f_{1}} i N_{k}\left(\frac{1-e^{-\lambda w_{k}}}{\lambda}\right)\left(\frac{w_{k}^{v}}{\Gamma(v+1)}\right)+\mathrm{七}_{f_{2}} \lambda N_{k}\left(\frac{1-e^{-\lambda w_{k}}}{\lambda}\right) \\
& \left.+N_{k} \mathrm{七}_{g k}, \text { such that } k=0,1, \ldots, m\right\} .
\end{aligned}
$$

Proof Consider the space of piecewise continuous functions

$$
V=\{\mu: J \rightarrow \mathbb{R} \text { such that } \mu \in P C(J, \mathbb{R})\},
$$


endowed with the generalized metric on $V$, defined by

$$
\begin{aligned}
d(\mu, v)= & \inf \left\{C_{1}+C_{2} \in[0,+\infty]\right. \text { such that } \\
& \left.|\mu(w)-v(w)| \leq\left(C_{1}+C_{2}\right)(\varphi(w)+\psi) \text { for all } w \in J\right\},
\end{aligned}
$$

where

$$
C_{1} \in\left\{C \in[0, \infty] \text { such that }|\mu(w)-v(w)| \leq C \varphi(w) \text { for all } w \in\left(w_{k}, s_{k}\right], k=0,1, \ldots, m\right\}
$$

and

$$
C_{2} \in\left\{C \in[0, \infty] \text { such that }|\mu(w)-v(w)| \leq C \psi \text { for all } w \in\left(s_{k-1}, w_{k}\right], k=1,2, \ldots, m\right\} .
$$

It is easy to verify that $(V, d)$ is a complete generalized metric space [80].

Define an operator $\Lambda: V \rightarrow V$ by

$$
(\Lambda z)(w)=\left\{\begin{array}{l}
\left\{\begin{array}{c}
\int_{0}^{w} e^{-\lambda(w-s)} I^{v} f\left(s, z(s),{ }^{c} \mathcal{D}^{v}(\mathcal{D}+\lambda) z(s)\right) d s \\
\quad+\frac{A_{11}}{\Gamma(p+1)} \int_{0}^{\eta}(\eta-s)^{p} e^{-\lambda(\eta-s)} I^{v} f\left(s, z(s),{ }^{c} \mathcal{D}^{v}(\mathcal{D}+\lambda) z(s)\right) d s \\
\quad-A_{11} \int_{0}^{T} e^{-\lambda(T-s)} I^{v} f\left(s, z(s),{ }^{c} \mathcal{D}^{v}(\mathcal{D}+\lambda) z(s)\right) d s \\
\quad+\left(A_{11}\left(\eta^{p} E_{(1, p+1)}(a w)-e^{\lambda T}\right)+e^{\lambda T}\right) z_{0}, \quad w \in\left(0, s_{0}\right],
\end{array}\right. \\
\left\{\begin{array}{l}
g_{k}(w, z(w)), \quad w \in\left(s_{k-1}, w_{k}\right], k=1,2, \ldots, m, \\
\quad+\frac{M_{k}}{\Gamma(p+1)} \int_{0}^{\eta}(\eta-s)^{p} e^{-\lambda(\eta-s)} I^{v} f(s, z(s)) d s \\
\quad-M_{k} \int_{0}^{T} e^{-\lambda(T-s)} I^{v} f\left(s, z(s),{ }^{c} \mathcal{D}^{v}(\mathcal{D}+\lambda) z(s)\right) d s \\
\quad+N_{k} \int_{0}^{w_{k}} e^{-\lambda\left(t_{k}-s\right)} I^{v} f\left(s, z(s),{ }^{c} \mathcal{D}^{v}(\mathcal{D}+\lambda) z(s)\right) d s \\
+N_{k} g_{k}\left(w_{k}, z\left(w_{k}\right)\right), \quad w \in\left(w_{k}, s_{k}\right], k=0,1, \ldots, m,
\end{array}\right.
\end{array}\right.
$$

for all $z$ belonging to $V$ and $w \in J$. Obviously, according to $\left(H_{1}\right), \Lambda$ is a well-defined operator.

Next we shall verify that $\Lambda$ is strictly contractive on $V$. Note that according to definition of $(V, d)$, for any $\mu, v \in V$, it is possible to find $C_{1}, C_{2} \in[0, \infty]$ such that

$$
|\mu(w)-v(w)| \leq\left\{\begin{array}{l}
C_{1} \varphi(w), \quad w \in\left(w_{k}, s_{k}\right], k=0, \ldots, m \\
C_{2} \psi, \quad w \in\left(s_{k-1}, w_{k}\right], k=1, \ldots, m
\end{array}\right.
$$

From the definition of $\Lambda$ in Eq. (4.7), $\left(H_{2}\right),\left(H_{3}\right)$, and (4.8), we obtain the following.

Case 1: For $w \in\left[0, s_{0}\right]$,

$$
\begin{aligned}
& |(\Lambda \mu)(w)-(\Lambda v)(w)| \\
& =\mid \int_{0}^{w} e^{-\lambda(w-s)} I^{v} f\left(s, \mu(s),{ }^{c} \mathcal{D}^{v}(\mathcal{D}+\lambda) \mu(s)\right) d s \\
& \quad-\int_{0}^{w} e^{-\lambda(w-s)} I^{v} f\left(s, v(s),{ }^{c} \mathcal{D}^{v}(\mathcal{D}+\lambda) v(s)\right) d s
\end{aligned}
$$




$$
\begin{aligned}
& +\frac{A_{11}}{\Gamma(p+1)} \int_{0}^{\eta}(\eta-s)^{p} e^{-\lambda(\eta-s)} I^{v} f\left(s, \mu(s),{ }^{c} \mathcal{D}^{v}(\mathcal{D}+\lambda) \mu(s)\right) d s \\
& -A_{11} \int_{0}^{T} e^{-\lambda(T-s)} I^{v} f\left(s, \mu(s),{ }^{c} \mathcal{D}^{\nu}(\mathcal{D}+\lambda) \mu(s)\right) d s \\
& +\left(A_{11}\left(\eta^{p} E_{(1, p+1)}(a w)-e^{\lambda T}\right)+e^{\lambda T}\right) z_{0} \\
& -\frac{A_{11}}{\Gamma(p+1)} \int_{0}^{\eta}(\eta-s)^{p} e^{-\lambda(\eta-s)} I^{v} f\left(s, v(s),{ }^{c} \mathcal{D}^{v}(\mathcal{D}+\lambda) v(s)\right) d s \\
& +A_{11} \int_{0}^{T} e^{-\lambda(T-s)} I^{v} f\left(s, v(s),{ }^{c} \mathcal{D}^{v}(\mathcal{D}+\lambda) v(s)\right) d s \\
& -\left(A_{11}\left(\eta^{p} E_{(1, p+1)}(a w)-e^{\lambda T}\right)+e^{\lambda T}\right) z_{0} \mid \\
& \leq \mid \int_{0}^{w} e^{-\lambda(w-s)} I^{v} f\left(s, \mu(s),{ }^{c} \mathcal{D}^{v}(\mathcal{D}+\lambda) \mu(s)\right) d s \\
& -\int_{0}^{w} e^{-\lambda(w-s)} I^{v} f\left(s, v(s),{ }^{c} \mathcal{D}^{v}(\mathcal{D}+\lambda) v(s)\right) d s \\
& +\mid \frac{A_{11}}{\Gamma(p+1)} \int_{0}^{\eta}(\eta-s)^{p} e^{-\lambda(\eta-s)} I^{v} f\left(s, \mu(s),{ }^{c} \mathcal{D}^{v}(\mathcal{D}+\lambda) \mu(s)\right) d s \\
& -\frac{A_{11}}{\Gamma(p+1)} \int_{0}^{\eta}(\eta-s)^{p} e^{-\lambda(\eta-s)} I^{v} f\left(s, v(s),{ }^{c} \mathcal{D}^{v}(\mathcal{D}+\lambda) v(s)\right) d s \\
& +\mid A_{11} \int_{0}^{T} e^{-\lambda(T-s)} I^{v} f\left(s, v(s),{ }^{c} \mathcal{D}^{v}(\mathcal{D}+\lambda) v(s)\right) d s \\
& -A_{11} \int_{0}^{T} e^{-\lambda(T-s)} I^{v} f\left(s, \mu(s),{ }^{c} \mathcal{D}^{v}(\mathcal{D}+\lambda) \mu(s)\right) d s \\
& \leq \int_{0}^{w} e^{-\lambda(w-s)} I^{v}\left|f\left(s, \mu(s),{ }^{c} \mathcal{D}^{v}(\mathcal{D}+\lambda) \mu(s)\right) d s-f\left(s, v(s),{ }^{c} \mathcal{D}^{v}(\mathcal{D}+\lambda) v(s)\right) d s\right| \\
& +A_{11} \int_{0}^{T} e^{-\lambda(T-s)} I^{v}\left|f\left(s, v(s),{ }^{c} \mathcal{D}^{v}(\mathcal{D}+\lambda) v(s)\right) d s-f\left(s, \mu(s),{ }^{c} \mathcal{D}^{v}(\mathcal{D}+\lambda) \mu(s)\right) d s\right| \\
& +\frac{A_{11}}{\Gamma(p+1)} \int_{0}^{\eta}(\eta-s)^{p} e^{-\lambda(\eta-s)} I^{\nu} \mid f\left(s, \mu(s),{ }^{c} \mathcal{D}^{\nu}(\mathcal{D}+\lambda) \mu(s)\right) d s \\
& -f\left(s, v(s),{ }^{c} \mathcal{D}^{v}(\mathcal{D}+\lambda) v(s)\right) d s \\
& \leq \succeq_{f_{1}} \int_{0}^{w} e^{-\lambda(w-s)} I^{v}|\mu(s)-v(s)| d s \\
& +\succeq_{f_{2}} \int_{0}^{w} e^{-\lambda(w-s)} I^{v}\left|{ }^{c} \mathcal{D}^{v}(\mathcal{D}+\lambda) \mu(s)-{ }^{c} \mathcal{D}^{v}(\mathcal{D}+\lambda) v(s)\right| d s \\
& +\succeq_{f_{1}} A_{11} \int_{0}^{T} e^{-\lambda(T-s)} I^{v}|v(s)-\mu(s)| d s \\
& +\mathrm{f}_{f_{1}} \frac{A_{11}}{\Gamma(p+1)} \int_{0}^{\eta}(\eta-s)^{p} e^{-\lambda(\eta-s)} I^{v}|\mu(s)-v(s)| d s \\
& +\mathrm{七}_{f_{2}} A_{11} \int_{0}^{T} e^{-\lambda(T-s)} I^{v}\left|{ }^{c} \mathcal{D}^{v}(\mathcal{D}+\lambda) v(s)-{ }^{c} \mathcal{D}^{v}(\mathcal{D}+\lambda) \mu(s)\right| d s
\end{aligned}
$$


Zada et al. Advances in Difference Equations

(2019) 2019:489

Page 13 of 26

$$
\begin{aligned}
& +\mathrm{七}_{f_{2}} \frac{A_{11}}{\Gamma(p+1)} \int_{0}^{\eta}(\eta-s)^{p} e^{-\lambda(\eta-s)} I^{v}\left|{ }^{c} \mathcal{D}^{v}(\mathcal{D}+\lambda) \mu(s)-{ }^{c} \mathcal{D}^{v}(\mathcal{D}+\lambda) v(s)\right| d s \\
& =\mathrm{七}_{f_{1}} \int_{0}^{w} e^{-\lambda(w-s)} I^{v}|\mu(s)-v(s)| d s+\mathrm{七}_{f_{2}} \int_{0}^{w} e^{-\lambda(w-s)} I^{v c} \mathcal{D}^{v}(\mathcal{D}+\lambda)|\mu(s)-v(s)| d s \\
& +\succeq_{f_{1}} A_{11} \int_{0}^{T} e^{-\lambda(T-s)} I^{v}|v(s)-\mu(s)| d s \\
& +\mathrm{Ł}_{f_{2}} A_{11} \int_{0}^{T} e^{-\lambda(T-s)} I^{\nu c} \mathcal{D}^{v}(\mathcal{D}+\lambda)|v(s)-\mu(s)| d s \\
& +\mathrm{七}_{f_{1}} \frac{A_{11}}{\Gamma(p+1)} \int_{0}^{\eta}(\eta-s)^{p} e^{-\lambda(\eta-s)} I^{v}|\mu(s)-v(s)| d s \\
& +\mathrm{七}_{f_{2}} \frac{A_{11}}{\Gamma(p+1)} \int_{0}^{\eta}(\eta-s)^{p} e^{-\lambda(\eta-s)} I^{\nu c} \mathcal{D}^{\nu}(\mathcal{D}+\lambda)|\mu(s)-v(s)| d s \\
& \leq \mathrm{七}_{f_{1}} C_{1} \int_{0}^{w} e^{-\lambda(w-s)} I^{v}|\varphi(s)| d s+\mathrm{七}_{f_{2}} C_{1} \int_{0}^{w} e^{-\lambda(w-s)} I^{\nu c} \mathcal{D}^{v}(\mathcal{D}+\lambda)|\varphi(s)| d s \\
& +\mathrm{Ł}_{f_{1}} C_{1} A_{11} \int_{0}^{T} e^{-\lambda(T-s)} I^{v}|\varphi(s)| d s+\mathrm{Ł}_{f_{2}} C_{1} A_{11} \int_{0}^{T} e^{-\lambda(T-s)} I^{\nu c} \mathcal{D}^{\nu}(\mathcal{D}+\lambda)|\varphi(s)| d s \\
& +\frac{A_{11} C_{1} \mathrm{Ł}_{f_{1}}}{\Gamma(p+1)} \int_{0}^{\eta}(\eta-s)^{p} e^{-\lambda(\eta-s)} I^{v}|\varphi(s)| d s \\
& +\frac{A_{11} C_{1} \mathrm{Ł}_{f_{2}}}{\Gamma(p+1)} \int_{0}^{\eta}(\eta-s)^{p} e^{-\lambda(\eta-s)} I^{\nu c} \mathcal{D}^{v}(\mathcal{D}+\lambda)|\varphi(s)| d s \\
& =\mathrm{Ł}_{f_{1}} C_{1} \int_{0}^{w} e^{-\lambda(w-s)} I^{v}|\varphi(s)| d s+\mathrm{七}_{f_{2}} C_{1} \int_{0}^{w} e^{-\lambda(w-s)}(\mathcal{D}+\lambda)|\varphi(s)| d s \\
& +\mathrm{Ł}_{f_{1}} C_{1} A_{11} \int_{0}^{T} e^{-\lambda(T-s)} I^{v}|\varphi(s)| d s+\succeq_{f_{2}} C_{1} A_{11} \int_{0}^{T} e^{-\lambda(T-s)}(\mathcal{D}+\lambda)|\varphi(s)| d s \\
& +\frac{A_{11} C_{1} \mathrm{Ł}_{f_{1}}}{\Gamma(p+1)} \int_{0}^{\eta}(\eta-s)^{p} e^{-\lambda(\eta-s)} I^{v}|\varphi(s)| d s \\
& +\frac{A_{11} C_{1} \mathrm{Ł}_{f_{2}}}{\Gamma(p+1)} \int_{0}^{\eta}(\eta-s)^{p} e^{-\lambda(\eta-s)}(\mathcal{D}+\lambda)|\varphi(s)| d s \\
& \leq \mathrm{七}_{f_{1}} C_{1}\left(\int_{0}^{w} e^{-\lambda(w-s)} d s\right)\left(\int_{0}^{w} I^{v}(\varphi(s)) d s\right) \\
& +\mathrm{七}_{f_{2}} C_{1}\left(\int_{0}^{w} e^{-\lambda(w-s)} d s\right)\left(\int_{0}^{w}(\mathcal{D}+\lambda)(\varphi(s)) d s\right) \\
& +\mathrm{Ł}_{f_{1}} C_{1} A_{11}\left(\int_{0}^{T} e^{-\lambda(T-s)} d s\right)\left(\int_{0}^{T} I^{v}(\varphi(s)) d s\right) \\
& +\mathrm{七}_{f_{2}} C_{1} A_{11}\left(\int_{0}^{T} e^{-\lambda(T-s)} d s\right)\left(\int_{0}^{T}(\mathcal{D}+\lambda)(\varphi(s)) d s\right) \\
& +\mathrm{七}_{f_{1}} C_{1} \frac{A_{11}}{\Gamma(p+1)}\left(\int_{0}^{\eta}(\eta-s)^{p} d s\right)\left(\int_{0}^{\eta} e^{-\lambda(\eta-s)} d s\right)\left(\int_{0}^{\eta} I^{\nu}(\varphi(s)) d s\right) \\
& +\mathrm{七}_{f_{2}} C_{1} \frac{A_{11}}{\Gamma(p+1)}\left(\int_{0}^{\eta}(\eta-s)^{p} d s\right)\left(\int_{0}^{\eta} e^{-\lambda(\eta-s)} d s\right)\left(\int_{0}^{\eta}(\mathcal{D}+\lambda)(\varphi(s)) d s\right) \\
& \leq \mathrm{七}_{f_{1}} C_{1}\left(\frac{1-e^{-\lambda w}}{\lambda}\right) C_{\varphi} \varphi(w)+\mathrm{七}_{f_{2}} C_{1}\left(\frac{1-e^{-\lambda w}}{\lambda}\right) C_{\gamma} \varphi(w)
\end{aligned}
$$




$$
\begin{aligned}
& +\mathrm{七}_{f_{1}} C_{1} A_{11}\left(\frac{1-e^{-\lambda T}}{\lambda}\right) C_{\varphi} \varphi(w)+\mathrm{七}_{f_{2}} C_{1} A_{11}\left(\frac{1-e^{-\lambda T}}{\lambda}\right) C_{\gamma} \varphi(w) \\
& +\mathrm{七}_{f_{1}} C_{1} \frac{A_{11}}{\Gamma(p+1)} \frac{\eta^{p+1}}{p+1}\left(\frac{1-e^{-\lambda \eta}}{\lambda}\right) C_{\varphi} \varphi(w) \\
& +\mathrm{七}_{f_{2}} C_{1} \frac{A_{11}}{\Gamma(p+1)} \frac{\eta^{p+1}}{p+1}\left(\frac{1-e^{-\lambda \eta}}{\lambda}\right) C_{\gamma} \varphi(w) \\
= & C_{1}\left(\frac{1-e^{-\lambda w}}{\lambda}\right) \varphi(w)\left(\mathrm{七}_{f_{1}} C_{\varphi}+\mathrm{七}_{f_{2}} C_{\gamma}\right)+C_{1} A_{11}\left(\frac{1-e^{-\lambda T}}{\lambda}\right) \varphi(w)\left(\mathrm{七}_{f_{1}} C_{\varphi}+\mathrm{七}_{f_{2}} C_{\gamma}\right) \\
& +C_{1} \frac{A_{11}}{\Gamma(p+1)} \frac{\eta^{p+1}}{p+1}\left(\frac{1-e^{-\lambda \eta}}{\lambda}\right) \varphi(w)\left(\mathrm{七}_{f_{1}} C_{\varphi}+\mathrm{七}_{f_{2}} C_{\gamma}\right) \\
= & \left(\left(\frac{1-e^{-\lambda w}}{\lambda}\right)+A_{11}\left(\frac{1-e^{-\lambda T}}{\lambda}\right)+\frac{A_{11}}{\Gamma(p+1)} \frac{\eta^{p+1}}{p+1}\left(\frac{1-e^{-\lambda \eta}}{\lambda}\right)\right) \\
& \times C_{1} \varphi(w)\left(\mathrm{七}_{f_{1}} C_{\varphi}+\mathrm{七}_{f_{2}} C_{\gamma}\right) .
\end{aligned}
$$

Case 2: For $w \in\left(s_{k-1}, w_{k}\right]$, we have

$$
|(\Lambda \mu)(w)-(\Lambda v)(w)|=\left|g_{k}(w, \mu(w))-g_{k}(w, v(w))\right| \leq \mathrm{七}_{g k}|\mu(w)-v(w)| \leq \mathrm{七}_{g k} C_{2} \psi .
$$

Case 3: For $w \in\left(w_{k}, s_{k}\right]$ and $s \in\left(w_{k}, s_{k}\right]$,

$$
\begin{aligned}
|(\Lambda \mu)(w)-(\Lambda v)(w)| & \mid \int_{0}^{w} e^{-\lambda(w-s)} I^{v} f\left(s, \mu(s),{ }^{c} \mathcal{D}^{v}(\mathcal{D}+\lambda) \mu(s)\right) d s \\
& -\int_{0}^{w} e^{-\lambda(w-s)} I^{v} f\left(s, v(s),{ }^{C} \mathcal{D}^{v}(\mathcal{D}+\lambda) v(s)\right) d s \\
& +\frac{M_{k}}{\Gamma(p+1)} \int_{0}^{\eta}(\eta-s)^{p} e^{-\lambda(\eta-s)} I^{v} f\left(s, \mu(s),{ }^{c} \mathcal{D}^{v}(\mathcal{D}+\lambda) \mu(s)\right) d s \\
& -M_{k} \int_{0}^{T} e^{-\lambda(T-s)} I^{v} f\left(s, \mu(s),{ }^{c} \mathcal{D}^{v}(\mathcal{D}+\lambda) \mu(s)\right) d s \\
& +N_{k} \int_{0}^{w_{k}} e^{-\lambda\left(w_{k}-s\right)} I^{v} f\left(s, \mu(s),{ }^{c} \mathcal{D}^{v}(\mathcal{D}+\lambda) \mu(s)\right) d s \\
& -\frac{M_{k}}{\Gamma(p+1)} \int_{0}^{\eta}(\eta-s)^{p} e^{-\lambda(\eta-s)} I^{v} f\left(s, v(s),{ }^{c} \mathcal{D}^{v}(\mathcal{D}+\lambda) v(s)\right) d s \\
& +M_{k} \int_{0}^{T} e^{-\lambda(T-s)} I^{v} f\left(s, v(s),{ }^{c} \mathcal{D}^{v}(\mathcal{D}+\lambda) v(s)\right) d s \\
& -N_{k} \int_{0}^{w_{k}} e^{-\lambda\left(w_{k}-s\right)} I^{v} f\left(s, v(s),{ }^{c} \mathcal{D}^{v}(\mathcal{D}+\lambda) v(s)\right) d s \\
& +N_{k} g_{k}\left(w_{k}, \mu\left(w_{k}\right)\right)-N_{k} g_{k}\left(w_{k}, v\left(w_{k}\right)\right) \mid \\
\leq & \int_{0}^{w} e^{-\lambda(w-s)} I^{v} \mid f\left(s, \mu(s),{ }^{c} \mathcal{D}^{v}(\mathcal{D}+\lambda) \mu(s)\right) \\
& -f\left(s, v(s),{ }^{c} \mathcal{D}^{v}(\mathcal{D}+\lambda) v(s)\right) \mid d s+N_{k} \mathrm{七}_{g k} C_{2} \psi \\
& +\frac{M_{k}}{\Gamma(p+1)} \int_{0}^{\eta}(\eta-s)^{p} e^{-\lambda(\eta-s)} I^{v} \mid f\left(s, \mu(s),{ }^{c} \mathcal{D}^{v}(\mathcal{D}+\lambda) \mu(s)\right) \\
&
\end{aligned}
$$




$$
\begin{aligned}
& -f\left(s, v(s),{ }^{c} \mathcal{D}^{v}(\mathcal{D}+\lambda) v(s)\right) \mid d s \\
& +M_{k} \int_{0}^{T} e^{-\lambda(T-s)} I^{v}\left|f\left(s, v(s),{ }^{c} \mathcal{D}^{v}(\mathcal{D}+\lambda) v(s)\right)-f\left(s, \mu(s),{ }^{c} \mathcal{D}^{v}(\mathcal{D}+\lambda) \mu(s)\right)\right| d s \\
& +N_{k} \int_{0}^{w_{k}} e^{-\lambda\left(w_{k}-s\right)} I^{v}\left|f\left(s, \mu(s),{ }^{c} \mathcal{D}^{v}(\mathcal{D}+\lambda) \mu(s)\right)-f\left(s, v(s),{ }^{c} \mathcal{D}^{v}(\mathcal{D}+\lambda) v(s)\right)\right| d s \\
& \leq \succeq_{f_{1}} \int_{0}^{w} e^{-\lambda(w-s)} I^{v}|\mu(s)-v(s)| d s \\
& +\mathrm{七}_{f_{2}} \int_{0}^{w} e^{-\lambda(w-s)} I^{v}\left|{ }^{c} \mathcal{D}^{v}(\mathcal{D}+\lambda) \mu(s)-{ }^{c} \mathcal{D}^{v}(\mathcal{D}+\lambda) v(s)\right| d s \\
& +\mathrm{七}_{f_{1}} \frac{M_{k}}{\Gamma(p+1)} \int_{0}^{\eta}(\eta-s)^{p} e^{-\lambda(\eta-s)} I^{v}|\mu(s)-v(s)| d s \\
& +\mathrm{七}_{f_{1}} M_{k} \int_{0}^{T} e^{-\lambda(T-s)} I^{v}|v(s)-\mu(s)| d s \\
& +\succeq_{f_{2}} \frac{M_{k}}{\Gamma(p+1)} \int_{0}^{\eta}(\eta-s)^{p} e^{-\lambda(\eta-s)} I^{v}\left|{ }^{c} \mathcal{D}^{v}(\mathcal{D}+\lambda) \mu(s)-{ }^{c} \mathcal{D}^{v}(\mathcal{D}+\lambda) v(s)\right| d s \\
& +\succeq_{f_{2}} M_{k} \int_{0}^{T} e^{-\lambda(T-s)} I^{v}\left|{ }^{c} \mathcal{D}^{v}(\mathcal{D}+\lambda) v(s)-{ }^{c} \mathcal{D}^{\nu}(\mathcal{D}+\lambda) \mu(s)\right| d s \\
& +\mathrm{七}_{f_{2}} N_{k} \int_{0}^{w_{k}} e^{-\lambda\left(w_{k}-s\right)} I^{v}\left|{ }^{c} \mathcal{D}^{v}(\mathcal{D}+\lambda) \mu(s)-{ }^{c} \mathcal{D}^{v}(\mathcal{D}+\lambda) v(s)\right| d s \\
& +\mathrm{七}_{f_{1}} N_{k} \int_{0}^{w_{k}} e^{-\lambda\left(w_{k}-s\right)} I^{v}|\mu(s)-v(s)| d s+N_{k} \mathrm{七}_{g k} C_{2} \psi \\
& =\mathrm{Ł}_{f_{1}} \int_{0}^{w} e^{-\lambda(w-s)} I^{v}|\mu(s)-v(s)| d s+\mathrm{Ł}_{f_{2}} \int_{0}^{w} e^{-\lambda(w-s)} I^{\nu c} \mathcal{D}^{v}(\mathcal{D}+\lambda)|\mu(s)-v(s)| d s \\
& +\mathrm{七}_{f_{1}} \frac{M_{k}}{\Gamma(p+1)} \int_{0}^{\eta}(\eta-s)^{p} e^{-\lambda(\eta-s)} I^{v}|\mu(s)-v(s)| d s \\
& +七_{f_{1}} N_{k} \int_{0}^{w_{k}} e^{-\lambda\left(w_{k}-s\right)} I^{v}|\mu(s)-v(s)| d s \\
& +\mathrm{七}_{f_{2}} \frac{M_{k}}{\Gamma(p+1)} \int_{0}^{\eta}(\eta-s)^{p} e^{-\lambda(\eta-s)} I^{\nu c} \mathcal{D}^{v}(\mathcal{D}+\lambda)|\mu(s)-v(s)| d s \\
& +\mathrm{七}_{f_{1}} M_{k} \int_{0}^{T} e^{-\lambda(T-s)} I^{v}|v(s)-\mu(s)| d s \\
& +\succeq_{f_{2}} M_{k} \int_{0}^{T} e^{-\lambda(T-s)} I^{v c} \mathcal{D}^{v}(\mathcal{D}+\lambda)|v(s)-\mu(s)| d s \\
& +\succeq_{f_{2}} N_{k} \int_{0}^{w_{k}} e^{-\lambda\left(w_{k}-s\right)} I^{v c} \mathcal{D}^{v}(\mathcal{D}+\lambda)|\mu(s)-v(s)| d s+N_{k} \succeq_{g k} C_{2} \psi \\
& \leq \mathrm{七}_{f_{1}} C_{1} \int_{0}^{w} e^{-\lambda(w-s)} I^{v}|\varphi(s)| d s+Ł_{f_{2}} C_{1} \int_{0}^{w} e^{-\lambda(w-s)}(\mathcal{D}+\lambda)|\varphi(s)| d s \\
& \left.+七_{f_{1}} C_{1} \frac{M_{k}}{\Gamma(p+1)} \int_{0}^{\eta}(\eta-s)^{p} e^{-\lambda(\eta-s)} I^{v} \mid \varphi(s)\right) \mid d s \\
& +\succeq_{f_{2}} C_{1} M_{k} \int_{0}^{T} e^{-\lambda(T-s)}(\mathcal{D}+\lambda)|\varphi(s)| d s \\
& +\succeq_{f_{2}} C_{1} \frac{M_{k}}{\Gamma(p+1)} \int_{0}^{\eta}(\eta-s)^{p} e^{-\lambda(\eta-s)}(\mathcal{D}+\lambda)|\varphi(s)| d s
\end{aligned}
$$


Zada et al. Advances in Difference Equations

(2019) 2019:489

Page 16 of 26

$$
\begin{aligned}
& +\mathrm{七}_{f_{1}} C_{1} M_{k} \int_{0}^{T} e^{-\lambda(T-s)} I^{v}|\varphi(s)| d s \\
& +\mathrm{七}_{f_{1}} C_{1} N_{k} \int_{0}^{w_{k}} e^{-\lambda\left(w_{k}-s\right)} I^{v}|\mu(s)-v(s)| d s \\
& +\mathrm{七}_{f_{2}} C_{1} N_{k} \int_{0}^{w_{k}} e^{-\lambda\left(w_{k}-s\right)}(\mathcal{D}+\lambda)|\varphi(s)| d s+N_{k} \mathrm{七}_{g k} C_{2} \psi \\
& \leq \mathrm{七}_{f_{1}} C_{1}\left(\int_{0}^{w} e^{-\lambda(w-s)} d s\right)\left(\int_{0}^{w} I^{v}(\varphi(s)) d s\right) \\
& +\mathrm{七}_{f_{2}} C_{1}\left(\int_{0}^{w} e^{-\lambda(w-s)} d s\right)\left(\int_{0}^{w}(\mathcal{D}+\lambda)(\varphi(s)) d s\right) \\
& +\mathrm{七}_{f_{1}} C_{1} \frac{M_{k}}{\Gamma(p+1)}\left(\int_{0}^{\eta}(\eta-s)^{p} d s\right)\left(\int_{0}^{\eta} e^{-\lambda(\eta-s)} d s\right)\left(\int_{0}^{\eta} I^{v}(\varphi(s)) d s\right) \\
& +\mathrm{七}_{f_{2}} C_{1} \frac{M_{k}}{\Gamma(p+1)}\left(\int_{0}^{\eta}(\eta-s)^{p} d s\right)\left(\int_{0}^{\eta} e^{-\lambda(\eta-s)} d s\right)\left(\int_{0}^{\eta}(\mathcal{D}+\lambda)(\varphi(s)) d s\right) \\
& +\mathrm{七}_{f_{1}} C_{1} M_{k}\left(\int_{0}^{T} e^{-\lambda(T-s)} d s\right)\left(\int_{0}^{T} I^{v}(\varphi(s)) d s\right) \\
& +\mathrm{七}_{f_{1}} C_{1} N_{k}\left(\int_{0}^{w_{k}} e^{-\lambda\left(w_{k}-s\right)} d s\right)\left(\int_{0}^{w_{k}} I^{v}(\varphi(s)) d s\right) \\
& +\mathrm{七}_{f_{2}} C_{1} M_{k}\left(\int_{0}^{T} e^{-\lambda(T-s)} d s\right)\left(\int_{0}^{T}(\mathcal{D}+\lambda)(\varphi(s)) d s\right) \\
& +\mathrm{七}_{f_{2}} C_{1} N_{k}\left(\int_{0}^{w_{k}} e^{-\lambda\left(w_{k}-s\right)} d s\right)\left(\int_{0}^{w_{k}}(\mathcal{D}+\lambda)(\varphi(s)) d s\right)+N_{k} \mathrm{七}_{g k} C_{2} \psi \\
& \leq \mathrm{七}_{f_{1}} C_{1}\left(\frac{1-e^{-\lambda w}}{\lambda}\right) C_{\varphi} \varphi(w)+\mathrm{七}_{f_{2}} C_{1}\left(\frac{1-e^{-\lambda w}}{\lambda}\right) C_{\gamma} \varphi(w) \\
& +\mathrm{七}_{f_{1}} C_{1} N_{k}\left(\frac{1-e^{-\lambda w_{k}}}{\lambda}\right) C_{\varphi} \varphi(w) \\
& +Ł_{f_{1}} C_{1} \frac{M_{k}}{\Gamma(p+1)} \frac{\eta^{p+1}}{p+1}\left(\frac{1-e^{-\lambda \eta}}{\lambda}\right) C_{\varphi} \varphi(w) \\
& +\mathrm{七}_{f_{2}} C_{1} \frac{M_{k}}{\Gamma(p+1)} \frac{\eta^{p+1}}{p+1}\left(\frac{1-e^{-\lambda \eta}}{\lambda}\right) C_{\gamma} \varphi(w) \\
& +\mathrm{七}_{f_{1}} C_{1} M_{k}\left(\frac{1-e^{-\lambda T}}{\lambda}\right) C_{\varphi} \varphi(w)+\succeq_{f_{2}} C_{1} M_{k}\left(\frac{1-e^{-\lambda T}}{\lambda}\right) C_{\gamma} \varphi(w) \\
& +\mathrm{七}_{f_{2}} C_{1} N_{k}\left(\frac{1-e^{-\lambda w_{k}}}{\lambda}\right) C_{\gamma} \varphi(w)+N_{k} \mathrm{七}_{g k} C_{2} \psi \\
& =C_{1}\left(\frac{1-e^{-\lambda w}}{\lambda}\right) \varphi(w)\left(\mathrm{E}_{f_{1}} C_{\varphi}+\mathrm{七}_{f_{2}} C_{\gamma}\right) \\
& +C_{1} \frac{M_{k}}{\Gamma(p+1)} \frac{\eta^{p+1}}{p+1}\left(\frac{1-e^{-\lambda \eta}}{\lambda}\right) \varphi(w)\left(\mathrm{七}_{f_{1}} C_{\varphi}+\mathrm{七}_{f_{2}} C_{\gamma}\right) \\
& +C_{1} M_{k}\left(\frac{1-e^{-\lambda T}}{\lambda}\right) \varphi(w)\left(\mathrm{Ł}_{f_{1}} C_{\varphi}+\mathrm{七}_{f_{2}} C_{\gamma}\right) \\
& +C_{1} N_{k}\left(\frac{1-e^{-\lambda w_{k}}}{\lambda}\right) \varphi(w)\left(\mathrm{Ł}_{f_{1}} C_{\varphi}+\succeq_{f_{2}} C_{\gamma}\right)+N_{k} \mathrm{七}_{g k} C_{2} \psi
\end{aligned}
$$




$$
\begin{aligned}
= & \left(\left(\frac{1-e^{-\lambda w}}{\lambda}\right)+\frac{M_{k}}{\Gamma(p+1)} \frac{\eta^{p+1}}{p+1}\left(\frac{1-e^{-\lambda \eta}}{\lambda}\right)+M_{k}\left(\frac{1-e^{-\lambda T}}{\lambda}\right)+N_{k}\left(\frac{1-e^{-\lambda w_{k}}}{\lambda}\right)\right) \\
& \times C_{1} \varphi(w)\left(\mathrm{七}_{f_{1}} C_{\varphi}+\mathrm{七}_{f_{2}} C_{\gamma}\right)+N_{k} \mathrm{七}_{g k} C_{2} \psi \\
\leq & \left\{\left(\left(\frac{1-e^{-\lambda w}}{\lambda}\right)+\frac{M_{k}}{\Gamma(p+1)} \frac{\eta^{p+1}}{p+1}\left(\frac{1-e^{-\lambda \eta}}{\lambda}\right)+M_{k}\left(\frac{1-e^{-\lambda T}}{\lambda}\right)\right.\right. \\
& \left.\left.+N_{k}\left(\frac{1-e^{-\lambda w_{k}}}{\lambda}\right)\right)\left(\mathrm{七}_{f_{1}} C_{\varphi}+\mathrm{七}_{f_{2}} C_{\gamma}\right)+N_{k} \mathrm{七}_{g k}\right\}\left(C_{1}+C_{2}\right)(\varphi(w)+\psi) .
\end{aligned}
$$

Also, for $w \in\left(w_{k}, s_{k}\right]$ and $s \in\left(s_{k-1}, w_{k}\right]$, we have

$$
\begin{aligned}
& |(\Lambda \mu)(w)-(\Lambda v)(w)| \\
& =\mid \int_{0}^{w} e^{-\lambda(w-s)} I^{v} f\left(s, \mu(s),{ }^{c} \mathcal{D}^{v}(\mathcal{D}+\lambda) \mu(s)\right) d s \\
& -\int_{0}^{w} e^{-\lambda(w-s)} I^{v} f\left(s, v(s),{ }^{c} \mathcal{D}^{v}(\mathcal{D}+\lambda) v(s)\right) d s \\
& +\frac{M_{k}}{\Gamma(p+1)} \int_{0}^{\eta}(\eta-s)^{p} e^{-\lambda(\eta-s)} I^{v} f\left(s, \mu(s),{ }^{c} \mathcal{D}^{v}(\mathcal{D}+\lambda) \mu(s)\right) d s \\
& -M_{k} \int_{0}^{T} e^{-\lambda(T-s)} I^{v} f\left(s, \mu(s),{ }^{c} \mathcal{D}^{v}(\mathcal{D}+\lambda) \mu(s)\right) d s \\
& +N_{k} \int_{0}^{w_{k}} e^{-\lambda\left(w_{k}-s\right)} I^{v} f\left(s, \mu(s),{ }^{c} \mathcal{D}^{v}(\mathcal{D}+\lambda) \mu(s)\right) d s \\
& -\frac{M_{k}}{\Gamma(p+1)} \int_{0}^{\eta}(\eta-s)^{p} e^{-\lambda(\eta-s)} I^{v} f\left(s, v(s),{ }^{c} \mathcal{D}^{v}(\mathcal{D}+\lambda) v(s)\right) d s \\
& +M_{k} \int_{0}^{T} e^{-\lambda(T-s)} I^{v} f\left(s, v(s),{ }^{c} \mathcal{D}^{v}(\mathcal{D}+\lambda) v(s)\right) d s \\
& -N_{k} \int_{0}^{w_{k}} e^{-\lambda\left(w_{k}-s\right)} I^{v} f\left(s, v(s),{ }^{c} \mathcal{D}^{v}(\mathcal{D}+\lambda) v(s)\right) d s \\
& +N_{k} g_{k}\left(w_{k}, \mu\left(w_{k}\right)\right)-N_{k} g_{k}\left(w_{k}, v\left(w_{k}\right)\right) \\
& \leq \mid \int_{0}^{w} e^{-\lambda(w-s)} I^{v} f\left(s, \mu(s),{ }^{c} \mathcal{D}^{v}(\mathcal{D}+\lambda) \mu(s)\right) d s \\
& -\int_{0}^{w} e^{-\lambda(w-s)} I^{v} f\left(s, v(s),{ }^{c} \mathcal{D}^{v}(\mathcal{D}+\lambda) v(s)\right) d s \\
& +\mid \frac{M_{k}}{\Gamma(p+1)} \int_{0}^{\eta}(\eta-s)^{p} e^{-\lambda(\eta-s)} I^{v} f\left(s, \mu(s),{ }^{c} \mathcal{D}^{v}(\mathcal{D}+\lambda) \mu(s)\right) d s \\
& -\frac{M_{k}}{\Gamma(p+1)} \int_{0}^{\eta}(\eta-s)^{p} e^{-\lambda(\eta-s)} I^{v} f\left(s, v(s),{ }^{c} \mathcal{D}^{v}(\mathcal{D}+\lambda) v(s)\right) d s \\
& +\mid M_{k} \int_{0}^{T} e^{-\lambda(T-s)} I^{v} f\left(s, v(s),{ }^{c} \mathcal{D}^{v}(\mathcal{D}+\lambda) v(s)\right) d s \\
& -M_{k} \int_{0}^{T} e^{-\lambda(T-s)} I^{v} f\left(s, \mu(s),{ }^{c} \mathcal{D}^{v}(\mathcal{D}+\lambda) \mu(s)\right) d s \\
& +\mid N_{k} \int_{0}^{w_{k}} e^{-\lambda\left(w_{k}-s\right)} I^{v} f\left(s, \mu(s),{ }^{c} \mathcal{D}^{v}(\mathcal{D}+\lambda) \mu(s)\right) d s
\end{aligned}
$$




$$
\begin{aligned}
& -N_{k} \int_{0}^{w_{k}} e^{-\lambda\left(w_{k}-s\right)} I^{v} f\left(s, v(s),{ }^{c} \mathcal{D}^{v}(\mathcal{D}+\lambda) v(s)\right) d s \\
& +\left|N_{k} g_{k}\left(w_{k}, \mu\left(w_{k}\right)\right)-N_{k} g_{k}\left(w_{k}, v\left(w_{k}\right)\right)\right| \\
& \leq \int_{0}^{w} e^{-\lambda(w-s)} I^{v}\left|f\left(s, \mu(s),{ }^{c} \mathcal{D}^{v}(\mathcal{D}+\lambda) \mu(s)\right)-f\left(s, v(s),{ }^{c} \mathcal{D}^{v}(\mathcal{D}+\lambda) v(s)\right)\right| d s \\
& +\frac{M_{k}}{\Gamma(p+1)} \int_{0}^{\eta}(\eta-s)^{p} e^{-\lambda(\eta-s)} I^{v} \mid f\left(s, \mu(s),{ }^{c} \mathcal{D}^{v}(\mathcal{D}+\lambda) \mu(s)\right) \\
& -f\left(s, v(s),{ }^{c} \mathcal{D}^{v}(\mathcal{D}+\lambda) v(s)\right) \mid d s \\
& +M_{k} \int_{0}^{T} e^{-\lambda(T-s)} I^{v}\left|f\left(s, v(s),{ }^{c} \mathcal{D}^{v}(\mathcal{D}+\lambda) v(s)\right)-f\left(s, \mu(s),{ }^{c} \mathcal{D}^{v}(\mathcal{D}+\lambda) \mu(s)\right)\right| d s \\
& +N_{k} \int_{0}^{w_{k}} e^{-\lambda\left(w_{k}-s\right)} I^{v}\left|f\left(s, \mu(s),{ }^{c} \mathcal{D}^{v}(\mathcal{D}+\lambda) \mu(s)\right)-f\left(s, v(s),{ }^{c} \mathcal{D}^{v}(\mathcal{D}+\lambda) v(s)\right)\right| d s \\
& +N_{k} \mathrm{七}_{g k} C_{2} \psi \\
& \leq \succeq_{f_{1}} \int_{0}^{w} e^{-\lambda(w-s)} I^{v}|\mu(s)-v(s)| d s \\
& +\mathrm{七}_{f_{1}} \frac{M_{k}}{\Gamma(p+1)} \int_{0}^{\eta}(\eta-s)^{p} e^{-\lambda(\eta-s)} I^{v}|\mu(s)-v(s)| d s \\
& +\succeq_{f_{2}} \int_{0}^{w} e^{-\lambda(w-s)} I^{v}\left|{ }^{c} \mathcal{D}^{v}(\mathcal{D}+\lambda) \mu(s)-{ }^{c} \mathcal{D}^{v}(\mathcal{D}+\lambda) v(s)\right| d s \\
& +\mathrm{七}_{f_{2}} \frac{M_{k}}{\Gamma(p+1)} \int_{0}^{\eta}(\eta-s)^{p} e^{-\lambda(\eta-s)} I^{v}\left|{ }^{c} \mathcal{D}^{v}(\mathcal{D}+\lambda) \mu(s)-{ }^{c} \mathcal{D}^{v}(\mathcal{D}+\lambda) v(s)\right| d s \\
& +\mathrm{七}_{f_{1}} M_{k} \int_{0}^{T} e^{-\lambda(T-s)} I^{v}|v(s)-\mu(s)| d s+\mathrm{七}_{f_{1}} N_{k} \int_{0}^{w_{k}} e^{-\lambda\left(w_{k}-s\right)} I^{v}|\mu(s)-v(s)| d s \\
& +七_{f_{2}} M_{k} \int_{0}^{T} e^{-\lambda(T-s)} I^{v}\left|c \mathcal{D}^{v}(\mathcal{D}+\lambda) v(s)-{ }^{c} \mathcal{D}^{v}(\mathcal{D}+\lambda) \mu(s)\right| d s \\
& +\succeq_{f_{2}} N_{k} \int_{0}^{w_{k}} e^{-\lambda\left(w_{k}-s\right)} I^{v}\left|{ }^{c} \mathcal{D}^{v}(\mathcal{D}+\lambda) \mu(s)-{ }^{c} \mathcal{D}^{v}(\mathcal{D}+\lambda) v(s)\right| d s+N_{k} \mathrm{七}_{g k} C_{2} \psi \\
& =\mathrm{Ł}_{f_{1}} \int_{0}^{w} e^{-\lambda(w-s)} I^{v}|\mu(s)-v(s)| d s+£_{f_{2}} \int_{0}^{w} e^{-\lambda(w-s)} I^{v c} \mathcal{D}^{v}(\mathcal{D}+\lambda)|\mu(s)-v(s)| d s \\
& +\succeq_{f_{1}} \frac{M_{k}}{\Gamma(p+1)} \int_{0}^{\eta}(\eta-s)^{p} e^{-\lambda(\eta-s)} I^{v}|\mu(s)-v(s)| d s+N_{k} \mathrm{七}_{g k} C_{2} \psi \\
& +七_{f_{2}} \frac{M_{k}}{\Gamma(p+1)} \int_{0}^{\eta}(\eta-s)^{p} e^{-\lambda(\eta-s)} I^{\nu c} \mathcal{D}^{\nu}(\mathcal{D}+\lambda)|\mu(s)-v(s)| d s \\
& +\mathrm{七}_{f_{1}} M_{k} \int_{0}^{T} e^{-\lambda(T-s)} I^{v}|v(s)-\mu(s)| d s \\
& +Ł_{f_{2}} M_{k} \int_{0}^{T} e^{-\lambda(T-s)} I^{v c} \mathcal{D}^{v}(\mathcal{D}+\lambda)|v(s)-\mu(s)| d s \\
& +\succeq_{f_{1}} N_{k} \int_{0}^{w_{k}} e^{-\lambda\left(w_{k}-s\right)} I^{v}|\mu(s)-v(s)| d s \\
& +\succeq_{f_{2}} N_{k} \int_{0}^{w_{k}} e^{-\lambda\left(w_{k}-s\right)} I^{v c} \mathcal{D}^{v}(\mathcal{D}+\lambda)|\mu(s)-v(s)| d s \\
& \leq \mathrm{七}_{f_{1}} C_{2} \psi \int_{0}^{w} e^{-\lambda(w-s)} I^{v}(1) d s+Ł_{f_{2}} C_{2} \psi \int_{0}^{w} e^{-\lambda(w-s)}(\mathcal{D}+\lambda)(1) d s
\end{aligned}
$$


Zada et al. Advances in Difference Equations

(2019) 2019:489

Page 19 of 26

$$
\begin{aligned}
& +\mathrm{七}_{f_{1}} C_{2} \psi \frac{M_{k}}{\Gamma(p+1)} \int_{0}^{\eta}(\eta-s)^{p} e^{-\lambda(\eta-s)} I^{v}(1) d s+\mathrm{七}_{f_{1}} C_{2} \psi M_{k} \int_{0}^{T} e^{-\lambda(T-s)} I^{v}(1) d s \\
& +\mathrm{七}_{f_{2}} C_{2} \psi \frac{M_{k}}{\Gamma(p+1)} \int_{0}^{\eta}(\eta-s)^{p} e^{-\lambda(\eta-s)}(\mathcal{D}+\lambda)(1) d s \\
& +\mathrm{七}_{f_{2}} C_{2} \psi M_{k} \int_{0}^{T} e^{-\lambda(T-s)}(\mathcal{D}+\lambda)(1) d s \\
& +\mathrm{七}_{f_{1}} C_{2} \psi N_{k} \int_{0}^{w_{k}} e^{-\lambda\left(w_{k}-s\right)} I^{v}(1) d s+\mathrm{七}_{f_{2}} C_{2} \psi N_{k} \int_{0}^{w_{k}} e^{-\lambda\left(w_{k}-s\right)}(\mathcal{D}+\lambda)(1) d s \\
& +N_{k} \succeq_{g k} C_{2} \psi \\
& =\mathrm{Ł}_{f_{1}} C_{2} \psi \int_{0}^{w} e^{-\lambda(w-s)} I^{\nu}(1) d s+\mathrm{Ł}_{f_{2}} C_{2} \psi \int_{0}^{w} e^{-\lambda(w-s)}(\mathcal{D}(1)+\lambda(1)) d s \\
& +\mathrm{七}_{f_{1}} C_{2} \psi \frac{M_{k}}{\Gamma(p+1)} \int_{0}^{\eta}(\eta-s)^{p} e^{-\lambda(\eta-s)} I^{v}(1) d s+\mathrm{七}_{f_{1}} C_{2} \psi M_{k} \int_{0}^{T} e^{-\lambda(T-s)} I^{v}(1) d s \\
& +\succeq_{f_{2}} C_{2} \psi \frac{M_{k}}{\Gamma(p+1)} \int_{0}^{\eta}(\eta-s)^{p} e^{-\lambda(\eta-s)}(\mathcal{D}(1)+\lambda(1)) d s \\
& +\mathrm{七}_{f_{2}} C_{2} \psi M_{k} \int_{0}^{T} e^{-\lambda(T-s)}(\mathcal{D}(1)+\lambda(1)) d s+\succeq_{f_{1}} C_{2} \psi N_{k} \int_{0}^{w_{k}} e^{-\lambda\left(w_{k}-s\right)} I^{v}(1) d s \\
& +\mathrm{七}_{f_{2}} C_{2} \psi N_{k} \int_{0}^{w_{k}} e^{-\lambda\left(w_{k}-s\right)}(\mathcal{D}(1)+\lambda(1)) d s+N_{k} \mathrm{七}_{g k} C_{2} \psi \\
& =\mathrm{七}_{f_{1}} C_{2} \psi \int_{0}^{w} e^{-\lambda(w-s)} I^{v}(1) d s \\
& +\mathrm{七}_{f_{2}} C_{2} \lambda \psi \int_{0}^{w} e^{-\lambda(w-s)} d s+\mathrm{七}_{f_{2}} C_{2} \psi \lambda N_{k} \int_{0}^{w_{k}} e^{-\lambda\left(w_{k}-s\right)} d s \\
& +\mathrm{七}_{f_{1}} C_{2} \psi \frac{M_{k}}{\Gamma(p+1)} \int_{0}^{\eta}(\eta-s)^{p} e^{-\lambda(\eta-s)} I^{v}(1) d s \\
& +\mathrm{七}_{f_{2}} C_{2} \psi \lambda \frac{M_{k}}{\Gamma(p+1)} \int_{0}^{\eta}(\eta-s)^{p} e^{-\lambda(\eta-s)} d s \\
& +\mathrm{Ł}_{f_{1}} C_{2} \psi M_{k} \int_{0}^{T} e^{-\lambda(T-s)} I^{\nu}(1) d s+\mathrm{Ł}_{f_{2}} C_{2} \psi \lambda M_{k} \int_{0}^{T} e^{-\lambda(T-s)} d s \\
& +\mathrm{七}_{f_{1}} C_{2} \psi N_{k} \int_{0}^{w_{k}} e^{-\lambda\left(w_{k}-s\right)} I^{v}(1) d s+N_{k} \mathrm{七}_{g k} C_{2} \psi \\
& \leq \mathrm{七}_{f_{1}} C_{2} \psi\left(\frac{1-e^{-\lambda w}}{\lambda}\right)\left(\frac{w^{v}}{\Gamma(v+1)}\right)+\mathrm{七}_{f_{2}} C_{2} \lambda \psi\left(\frac{1-e^{-\lambda w}}{\lambda}\right) \\
& +\mathrm{七}_{f_{2}} C_{2} \lambda \psi N_{k}\left(\frac{1-e^{-\lambda w_{k}}}{\lambda}\right) \\
& +\mathrm{七}_{f_{1}} C_{2} \psi \frac{M_{k}}{\Gamma(p+1)}\left(\frac{\eta^{p+1}}{p+1}\right)\left(\frac{1-e^{-\lambda \eta}}{\lambda}\right)\left(\frac{\eta^{\nu}}{\Gamma(v+1)}\right)+\mathrm{七}_{f_{2}} C_{2} \lambda \psi M_{k}\left(\frac{1-e^{-\lambda T}}{\lambda}\right) \\
& +\mathrm{七}_{f_{2}} C_{2} \lambda \psi \frac{M_{k}}{\Gamma(p+1)}\left(\frac{\eta^{p+1}}{p+1}\right)\left(\frac{1-e^{-\lambda \eta}}{\lambda}\right)+\mathrm{七}_{f_{1}} C_{2} \psi M_{k}\left(\frac{1-e^{-\lambda T}}{\lambda}\right)\left(\frac{T^{v}}{\Gamma(v+1)}\right) \\
& +\mathrm{七}_{f_{1}} C_{2} \psi N_{k}\left(\frac{1-e^{-\lambda w_{k}}}{\lambda}\right)\left(\frac{w_{k}^{v}}{\Gamma(v+1)}\right)+N_{k} \mathrm{七}_{g k} C_{2} \psi \\
& =\left\{\mathrm{Ł}_{f_{1}}\left(\frac{1-e^{-\lambda w}}{\lambda}\right)\left(\frac{w^{v}}{\Gamma(v+1)}\right)+\mathrm{Ł}_{f_{2}} \lambda\left(\frac{1-e^{-\lambda w}}{\lambda}\right)\right.
\end{aligned}
$$




$$
\begin{aligned}
& +七_{f_{2}} \lambda \frac{M_{k}}{\Gamma(p+1)}\left(\frac{\eta^{p+1}}{p+1}\right)\left(\frac{1-e^{-\lambda \eta}}{\lambda}\right) \\
& +Ł_{f_{1}} \frac{M_{k}}{\Gamma(p+1)}\left(\frac{\eta^{p+1}}{p+1}\right)\left(\frac{1-e^{-\lambda \eta}}{\lambda}\right)\left(\frac{\eta^{\nu}}{\Gamma(v+1)}\right) \\
& +\mathrm{七}_{f_{1}} M_{k}\left(\frac{1-e^{-\lambda T}}{\lambda}\right)\left(\frac{T^{v}}{\Gamma(v+1)}\right) \\
& +\mathrm{七}_{f_{2}} \lambda M_{k}\left(\frac{1-e^{-\lambda T}}{\lambda}\right)+\mathrm{七}_{f_{1}} N_{k}\left(\frac{1-e^{-\lambda w_{k}}}{\lambda}\right)\left(\frac{w_{k}^{v}}{\Gamma(v+1)}\right) \\
& \left.+\mathrm{七}_{f_{2}} \lambda N_{k}\left(\frac{1-e^{-\lambda w_{k}}}{\lambda}\right)+N_{k} \mathrm{七}_{g k}\right\} C_{2} \psi \\
& \leq\left\{\succeq_{f_{1}}\left(\frac{1-e^{-\lambda w}}{\lambda}\right)\left(\frac{w^{v}}{\Gamma(v+1)}\right)+\succeq_{f_{2}} \lambda\left(\frac{1-e^{-\lambda w}}{\lambda}\right)\right. \\
& +\mathrm{七}_{f_{2}} \lambda \frac{M_{k}}{\Gamma(p+1)}\left(\frac{\eta^{p+1}}{p+1}\right)\left(\frac{1-e^{-\lambda \eta}}{\lambda}\right) \\
& +\mathrm{七}_{f_{1}} \frac{M_{k}}{\Gamma(p+1)}\left(\frac{\eta^{p+1}}{p+1}\right)\left(\frac{1-e^{-\lambda \eta}}{\lambda}\right)\left(\frac{\eta^{v}}{\Gamma(v+1)}\right) \\
& +\succeq_{f_{1}} M_{k}\left(\frac{1-e^{-\lambda T}}{\lambda}\right)\left(\frac{T^{v}}{\Gamma(v+1)}\right) \\
& +\mathrm{七}_{f_{2}} \lambda M_{k}\left(\frac{1-e^{-\lambda T}}{\lambda}\right)+\mathrm{七}_{f_{1}} i N_{k}\left(\frac{1-e^{-\lambda w_{k}}}{\lambda}\right)\left(\frac{w_{k}^{\nu}}{\Gamma(v+1)}\right) \\
& \left.+\mathrm{七}_{f_{2}} \lambda N_{k}\left(\frac{1-e^{-\lambda w_{k}}}{\lambda}\right)+N_{k} \mathrm{七}_{g k}\right\}\left(C_{1}+C_{2}\right)(\varphi(w)+\psi) .
\end{aligned}
$$

From the above we have

$$
|(\Lambda \mu)(w)-(\Lambda v)(w)| \leq €\left(C_{1}+C_{2}\right)(\varphi(w)+\psi), \quad w \in[0, \tau]
$$

that is,

$$
d(\Lambda \mu, \Lambda v) \leq \succeq\left(C_{1}+C_{2}\right)(\varphi(w)+\psi)
$$

Hence, we conclude that

$$
d(\Lambda \mu, \Lambda v) \leq \succeq d(\mu, v)
$$

for any $\mu, v \in V$, since condition (4.5) is strictly contractive property is shown.

Now we take $\mu_{0} \in V$. From the piecewise continuous property of $\mu_{0}$ and $\Lambda \mu_{0}$, it follows that there exists a constant $0<G_{1}<\infty$ such that

$$
\begin{aligned}
\left|\left(\Lambda \mu_{0}\right)(w)-\mu_{0}(w)\right| \leq & \mid \int_{0}^{w} e^{-\lambda(w-s)} I^{v} f\left(s, \mu_{0}(s),{ }^{c} \mathcal{D}^{v}(\mathcal{D}+\lambda) \mu_{0}(s)\right) d s \\
& +\frac{A_{11}}{\Gamma(p+1)} \int_{0}^{\eta}(\eta-s)^{p} e^{-\lambda(\eta-s)} I^{v} f\left(s, \mu_{0}(s),{ }^{c} \mathcal{D}^{v}(\mathcal{D}+\lambda) \mu_{0}(s)\right) d s \\
& -A_{11} \int_{0}^{T} e^{-\lambda(T-s)} I^{v} f\left(s, \mu_{0}(s),{ }^{c} \mathcal{D}^{v}(\mathcal{D}+\lambda) \mu_{0}(s)\right) d s
\end{aligned}
$$




$$
\begin{aligned}
& +\left(A_{11}\left(\eta^{p} E_{(1, p+1)}(a w)-e^{\lambda T}\right)+e^{\lambda T}\right) z_{0}-\mu_{0}(w) \\
\leq & G_{1} \varphi(w) \leq G_{1}(\varphi(w)+\psi), \quad w \in\left(0, s_{0}\right] .
\end{aligned}
$$

There exists a constant $0<G_{2}<\infty$ such that

$$
\begin{aligned}
& \left|\left(\Lambda \mu_{0}\right)(w)-\mu_{0}(w)\right|=\left|g_{k}\left(w, \mu_{0}(w)\right)-\mu_{0}(w)\right| \leq G_{2} \psi \leq G_{2}(\varphi(w)+\psi), \\
& \quad w \in\left(s_{k-1}, w_{k}\right], k=1,2, \ldots, m .
\end{aligned}
$$

Also we can find a constant $0<G_{3}<\infty$ such that

$$
\begin{aligned}
\left|\left(\Lambda \mu_{0}\right)(w)-\mu_{0}(w)\right| \leq & \mid \int_{0}^{w} e^{-\lambda(w-s)} I^{v} f\left(s, \mu_{0}(s),{ }^{c} \mathcal{D}^{v}(\mathcal{D}+\lambda) \mu_{0}(s)\right) d s \\
& +\frac{M_{k}}{\Gamma(p+1)} \int_{0}^{\eta}(\eta-s)^{p} e^{-\lambda(\eta-s)} I^{v} f\left(s, \mu_{0}(s),{ }^{c} \mathcal{D}^{v}(\mathcal{D}+\lambda) \mu_{0}(s)\right) d s \\
& -M_{k} \int_{0}^{T} e^{-\lambda(T-s)} I^{v} f\left(s, \mu_{0}(s),{ }^{c} \mathcal{D}^{v}(\mathcal{D}+\lambda) \mu_{0}(s)\right) d s \\
& +N_{k} \int_{0}^{w_{k}} e^{-\lambda\left(w_{k}-s\right)} I^{v} f\left(s, \mu_{0}(s),{ }^{c} \mathcal{D}^{v}(\mathcal{D}+\lambda) \mu_{0}(s)\right) d s \\
& +N_{k} g_{k}\left(w_{k}, \mu_{0}\left(w_{k}\right)\right)-\mu_{0}(w) \mid \\
\leq & G_{3} \varphi(w) \leq G_{3}(\varphi(w)+\psi), \quad w \in\left(w_{k}, s_{k}\right], k=1,2, \ldots, m .
\end{aligned}
$$

Since $f, g_{k}$, and $\mu_{0}$ are bounded on $J$ and $\varphi()>$.0 , thus (4.6) implies that $d\left(\Lambda \mu_{0}, \mu_{0}\right)<\infty$.

By using the Banach fixed point theorem, there exists a continuous function $z: J \rightarrow \mathbb{R}$ such that $\Lambda^{n} \mu_{0} \rightarrow z_{0}$ in $(V, d)$ as $n \rightarrow \infty$ and $\Lambda z=z_{0}$, that is, $z_{0}$ satisfies Eq. (4.3) for every $w \in J$.

Now we show that $\left\{\mu \in V\right.$ such that $\left.d\left(\mu_{0}, \mu\right)<\infty\right\}=V$. For any $g \in V$, since $\mu$ and $\mu_{0}$ are bounded on $J$ and $\min _{w \in J}(\varphi(w)+\psi)>0$, there exists a constant $0<C_{\mu}<\infty$ such that $\left|\mu_{0}(w)-\mu(w)\right| \leq C_{\mu}(\varphi(w)+\psi)$ for any $w \in J$. Hence, we have $d\left(\mu_{0}, \mu\right)<\infty$ for all $\mu \in$ $V$, that is, $\left\{\mu \in V\right.$ such that $\left.d\left(\mu_{0}, \mu\right)<\infty\right\}=V$. Thus, we determine that $z$ is the unique continuous function with Eq. (4.3). From (3.2) and $\left(H_{4}\right)$, we can write

$$
\begin{aligned}
d(z, \Lambda z) \leq & \left(\left(\frac{1-e^{-\lambda w}}{\lambda}\right)+\frac{M_{k}}{\Gamma(p+1)} \frac{\eta^{p+1}}{p+1}\left(\frac{1-e^{-\lambda \eta}}{\lambda}\right)\right. \\
& \left.+M_{k}\left(\frac{1-e^{-\lambda T}}{\lambda}\right)+N_{k}\left(\frac{1-e^{-\lambda w_{k}}}{\lambda}\right)\right)\left(\mathrm{七}_{f_{1}} C_{\varphi}+\mathrm{Ł}_{f_{2}} C_{\gamma}\right)+N_{k} \mathrm{七}_{g k} .
\end{aligned}
$$

Summarizing, we have

$$
\begin{aligned}
d\left(z_{0}, z\right) \leq & \frac{d(\Lambda z, z)}{1-\succeq} \\
\leq & \left\{\left(\left(\frac{1-e^{-\lambda w}}{\lambda}\right)+\frac{M_{k}}{\Gamma(p+1)} \frac{\eta^{p+1}}{p+1}\left(\frac{1-e^{-\lambda \eta}}{\lambda}\right)\right.\right. \\
& \left.\left.+M_{k}\left(\frac{1-e^{-\lambda T}}{\lambda}\right)+N_{k}\left(\frac{1-e^{-\lambda w_{k}}}{\lambda}\right)\right)\left(\mathrm{七}_{f_{1}} C_{\varphi}+\mathrm{七}_{f_{2}} C_{\gamma}\right)+N_{k} \mathrm{七}_{g k}\right\}\left(\frac{1}{1-\mathrm{七}}\right) .
\end{aligned}
$$


This shows that (4.4) is true for $w \in J$.

Finally, we give an example to illustrate our main results.

\section{Example 4.4}

$$
\left\{\begin{array}{l}
{ }^{c} \mathcal{D}_{0, w}^{\frac{1}{2}}(\mathcal{D}+2) z(w)=\frac{|z(w)|+{ }^{c} \mathcal{D}_{0, w}^{\frac{1}{2}}(\mathcal{D}+2) z(w)}{8+e^{w}+w^{2}+{ }^{c} \mathcal{D}_{0, w}^{\frac{1}{2}}(\mathcal{D}+2) z(w)}, \quad w \in(0,1] \cup(2,3], \\
z(w)=\frac{z(w)}{\left(3+w^{2}\right)(1+|z(w)|)}, \quad w \in(1,2], \\
z(0)=\frac{\sqrt{2}}{3}, \quad z(1)=\frac{5}{6} \int_{0}^{\frac{1}{4}} \frac{\left(\frac{1}{4}-s\right)}{\Gamma \frac{4}{3}} d s, \quad 0<\eta<1,
\end{array}\right.
$$

and

$$
\left\{\begin{array}{l}
\left|{ }^{c} \mathcal{D}_{0, w}^{\frac{1}{2}}(\mathcal{D}+2) z(w)-\frac{|z(w)|+{ }^{c} \mathcal{D}_{0, w}^{\frac{1}{2}}(\mathcal{D}+2) z(w)}{8+e^{w}+w^{2}+{ }^{c} \mathcal{D}_{0, w}^{\frac{1}{2}}(\mathcal{D}+2) z(w)}\right| \leq e^{w}, \quad w \in(0,1] \cup(2,3] \\
\left|z(w)-\frac{z(w)}{\left(3+w^{2}\right)(1+|z(w)|)}\right| \leq 1, \quad w \in(1,2]
\end{array}\right.
$$

Let $J=[0,3], v=\frac{1}{2}, p=\frac{4}{3}, \eta=\frac{1}{4}$, and $0=w_{0}<s_{0}=1<w_{1}=2<s_{1}=\tau=T=3$. Denote $f(w, z(w))=\frac{|z(w)|}{8+e^{w}+w^{2}}$ with $\mathrm{七}_{f_{1}}=\frac{1}{4}, \mathrm{七}_{f_{2}}=\frac{1}{3}$ for $w \in(0,1] \cup(2,3]$ and $g_{1}(w, z(w))=\frac{z(w)}{\left(3+w^{2}\right)(1+|z(w)|)}$ with $L_{g_{k}}=1$ for $w \in(1,2]$. Putting $Ł_{f}=\frac{1}{4}, \varphi(w)=e^{w}$, and $C_{1}=C_{\varphi}=C_{\gamma}=1$, we have $\int_{0}^{w} I^{\frac{1}{2}} e^{s} d s \leq e^{w}$ and $七_{1} \approx 0.1231, \mathrm{七}_{2} \approx 0.4741$, so $七 \approx 0.4741<1$.

By Theorem 4.3, there exists a unique solution $z:[0,3] \rightarrow \mathbb{R}$ such that

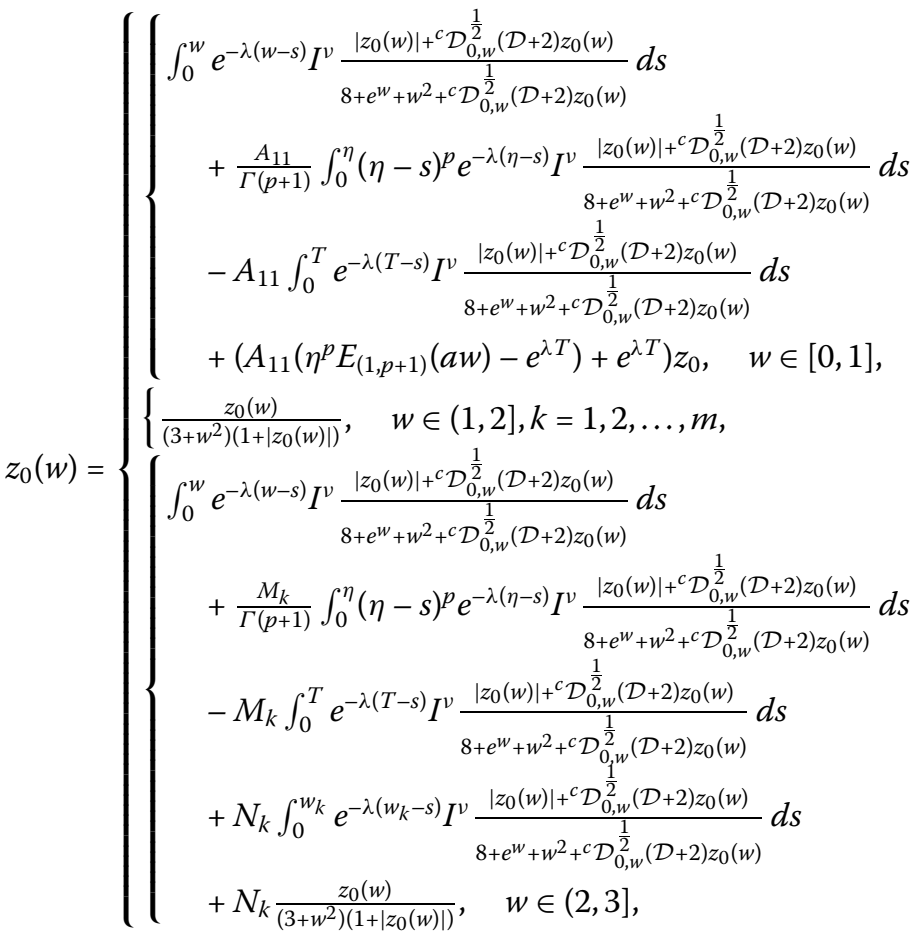

$$
\begin{aligned}
& \left|z(w)-z_{0}(w)\right| \leq\left\{\left(\left(\frac{1-e^{-\lambda w}}{\lambda}\right)+\frac{M_{k}}{\Gamma(p+1)} \frac{\eta^{p+1}}{p+1}\left(\frac{1-e^{-\lambda \eta}}{\lambda}\right)+M_{k}\left(\frac{1-e^{-\lambda T}}{\lambda}\right)\right.\right. \\
& \left.\left.+N_{k}\left(\frac{1-e^{-\lambda w_{k}}}{\lambda}\right)\right)\left(\succeq_{f_{1}} C_{\varphi}+\succeq_{f_{2}} C_{\gamma}\right)+N_{k} \succeq_{g k}\right\}\left(\frac{\varphi(w)+\psi}{1-\succeq}\right) \text {. }
\end{aligned}
$$


Putting maximum of $w=w_{k}=T=\tau$, we obtain

$$
\begin{aligned}
\left|z(w)-z_{0}(w)\right| \leq & \left\{\left(\left(\frac{1-e^{-\lambda \tau}}{\lambda}\right)+\frac{M_{k}}{\Gamma(p+1)} \frac{\eta^{p+1}}{p+1}\left(\frac{1-e^{-\lambda \eta}}{\lambda}\right)+M_{k}\left(\frac{1-e^{-\lambda \tau}}{\lambda}\right)\right.\right. \\
& \left.\left.+N_{k}\left(\frac{1-e^{-\lambda \tau}}{\lambda}\right)\right)\left(\mathrm{七}_{f_{1}} C_{\varphi}+\mathrm{七}_{f_{2}} C_{\gamma}\right)+N_{k} \mathrm{七}_{g k}\right\}\left(\frac{\varphi(w)+\psi}{1-€}\right) .
\end{aligned}
$$

Now, putting the values, we get

$$
\begin{aligned}
\left|z(w)-z_{0}(w)\right| & \leq 0.1059\left(\frac{e^{w}+1}{1-0.4741}\right) \\
& \leq 0.20136\left(e^{w}+1\right) \quad \text { for all } w \in[0,3]
\end{aligned}
$$

Thus problem (4.9) is Ulam-Hyers-Rassias stable.

\section{Conclusion}

In this article, we considered a nonlocal boundary value problem of nonlinear implicit impulsive Langevin equations with mixed derivatives and presented its Ulam-Hyers-Rassias stability. After introduction, we built a uniform structure to originate a formula of solutions for our proposed model. We implemented the new concept of generalized UlamHyers-Rassias stability to our proposed model; finally we solved a particular example for our proposed model.

\section{Acknowledgements}

Not applicable.

\section{Funding}

This work is supported by the National Natural Science Foundation of China (Grant No. 11601048), Technology Research Foundation of Chongqing Educational Committee (Grant No. KJQN201900539), Natural Science Foundation of Chongqing Normal University (Grant No. 16XYY24).

\section{Competing interests}

All authors declare that they have no conflict of interests.

Authors' contributions

All the authors have equally contributed to this manuscript. All authors read and approved the final manuscript.

\section{Author details}

${ }^{1}$ Department of Mathematics, University of Peshawar, Peshawar, Pakistan. ${ }^{2}$ School of Mathematical Sciences, Chongqing Normal University, Chongaing, China. ${ }^{3}$ College of Mathematics and System Sciences, Shandong University of Science and Technology, Qingdao, China.

\section{Publisher's Note}

Springer Nature remains neutral with regard to jurisdictional claims in published maps and institutional affiliations.

Received: 8 September 2019 Accepted: 6 November 2019 Published online: 28 November 2019

\section{References}

1. Rizwan, R., Zada, A., Wang, X.: Stability analysis of nonlinear implicit fractional Langevin equation with non-instantaneous impulses. Adv. Differ. Equ. 2019, Article ID 85 (2019)

2. Rizwan, R., Zada, A.: Nonlinear impulsive Langevin equation with mixed derivatives. Math. Methods Appl. Sci. (2019). https://doi.org/10.1002/mma.5902

3. Mainardi, F., Pironi, P.: The fractional Langevin equation: Brownian motion revisited. Extr. Math. 11(1), 140-154 (1996)

4. Zhai, C., Li, P., Li, H.: Single upper-solution or lower-solution method for Langevin equations with two fractional orders. Adv. Differ. Equ. 2018, Article ID 360 (2018)

5. Kilbas, A.A., Srivastava, H.M., Trujillo, J.J.: Theory and Applications of Fractional Differential Equation. Elsevier, Amsterdam (2006)

6. Podlubny, l.: Fractional Differential Equations. Academic Press, Sun Diego (1999) 
7. Tarasov, V.E.: Fractional Dynamics: Application of Fractional Calculus to Dynamics of Particles, Fields and Media. Springer, Berlin (2011)

8. Baleanu, D., Diethelm, K., Scalas, E., Trujillo, J.J.: Fractional Calculus Models and Numerical Methods, Series on Complexity, Nonlinearity and Chaos. World Scientific, Singapore (2012)

9. Tang, S., Zada, A., Faisal, S., El-Sheikh, M.M.A., Li, T.: Stability of higher-order nonlinear impulsive differential equations. J. Nonlinear Sci. Appl. 9, 4713-4721 (2016)

10. Zada, A., Ali, S.: Stability of integral Caputo-type boundary value problem with noninstantaneous impulses. Int. J. Appl. Comput. Math. 5, 55 (2019)

11. Yue, Z., Zou, Y.: New uniqueness results for fractional differential equation with dependence on the first order derivative. Adv. Differ. Equ. 2019, Article ID 38 (2019)

12. Cui, Y.: Uniqueness of solution for boundary value problems for fractional differential equations. Appl. Math. Lett. 51 48-54 (2016)

13. Cui, Y., Ma, W., Sun, Q., Su, X.: New uniqueness results for boundary value problem of fractional differential equation. Nonlinear Anal., Model. Control 23(1), 31-39 (2018)

14. Zou, Y., He, G.: On the uniqueness of solutions for a class of fractional differential equations. Appl. Math. Lett. 74 68-73 (2017)

15. Zhang, X., Wu, J., Liu, L., Wu, Y., Cui, Y.: Convergence analysis of iterative scheme and error estimation of positive solution for a fractional differential equation. Math. Model. Anal. 23(4), 611-626 (2018)

16. He, J., Zhang, X., Liu, L., Wu, Y., Cui, Y.: Existence and asymptotic analysis of positive solutions for a singular fractional differential equation with nonlocal boundary conditions. Bound. Value Probl. 2018, Article ID 189 (2018)

17. Zhang, X., Liu, L., Wu, Y., Zou, Y.: Existence and uniqueness of solutions for systems of fractional differential equations with Riemann-Stieltjes integral boundary condition. Adv. Differ. Equ. 2018, Article ID 204 (2018)

18. Zhong, Q., Zhang, X., Lu, X., Fu, Z.: Uniqueness of successive positive solution for nonlocal singular higher-order fractional differential equations involving arbitrary derivatives. J. Funct. Spaces 2018, Article ID 6207682 (2018)

19. Sheng, K., Zhang, W., Bai, Z.: Positive solutions to fractional boundary-value problems with $p$-Laplacian on time scales. Bound. Value Probl. 2018, Article ID 70 (2018)

20. Hao, X., Wang, H., Liu, L., Cui, Y.: Positive solutions for a system of nonlinear fractional nonlocal boundary value problems with parameters and p-Laplacian operator. Bound. Value Probl. 2017, Article ID 182 (2017)

21. Dong, X., Bai, Z., Zhang, S.: Positive solutions to boundary value problems of $p$-Laplacian with fractional derivative. Bound. Value Probl. 2017, Article ID 5 (2017)

22. Tian, Y., Sun, S., Bai, Z:: Positive solutions of fractional differential equations with $p$-Laplacian. J. Funct. Spaces 2017 Article ID 3187492 (2017)

23. Zou, Y: Positive solutions for a fractional boundary value problem with a perturbation term. J. Funct. Spaces 2018 Article ID 9070247 (2018)

24. Sun, Q., Ji, H., Cui, Y.: Positive solutions for boundary value problems of fractional differential equation with integral boundary conditions. J. Funct. Spaces 2018, Article ID 6461930 (2018)

25. Song, Q., Bai, Z:: Positive solutions of fractional differential equations involving the Riemann-Stieltjes integral boundary condition. Adv. Differ. Equ. 2018, Article ID 183 (2018)

26. Cheng, W., Xu, J., Cui, Y.: Positive solutions for a system of nonlinear semipositone fractional $q$-difference equations with q-integral boundary conditions. J. Nonlinear Sci. Appl. 10(8), 4430-4440 (2017)

27. Qiu, X., Xu, J., O'Regan, D., Cui, Y.: Positive solutions for a system of nonlinear semipositone boundary value problems with Riemann-Liouville fractional derivatives. J. Funct. Spaces 2018, Article ID 7351653 (2018)

28. Chen, C., Xu, J., O'Regan, D., Fu, Z.: Positive solutions for a system of semipositone fractional difference boundary value problems. J. Funct. Spaces 2018, Article ID 6835028 (2018)

29. Li, H., Zhang, J.: Positive solutions for a system of fractional differential equations with two parameters. J. Funct. Spaces 2018, Article ID 1462505 (2018)

30. Sun, Q., Meng, S., Cui, Y.: Existence results for fractional order differential equation with nonlocal Erdélyi-Kober and generalized Riemann-Liouville type integral boundary conditions at resonance. Adv. Differ. Equ. 2018, Article ID 243 (2018)

31. Zou, Y., He, G.: The existence of solutions to integral boundary value problems of fractional differential equations at resonance. J. Funct. Spaces 2017, Article ID 2785937 (2017)

32. Ma, W., Meng, S., Cui, Y.: Resonant integral boundary value problems for Caputo fractional differential equations. Math. Probl. Eng. 2018, Article ID 5438592 (2018)

33. Zhang, Y.: Existence results for a coupled system of nonlinear fractional multi-point boundary value problems at resonance. J. Inequal. Appl. 2018, Article ID 198 (2018)

34. He, L., Dong, X., Bai, Z., Chen, B.: Solvability of some two-point fractional boundary value problems under barrier strip conditions. J. Funct. Spaces 2017, Article ID 1465623 (2017)

35. Song, Q., Dong, X., Bai, Z., Chen, B.: Existence for fractional Dirichlet boundary value problem under barrier strip conditions. J. Nonlinear Sci. Appl. 10, 3592-3598 (2017)

36. Wu, J., Zhang, X., Liu, L., Wu, Y., Cui, Y.: The convergence analysis and error estimation for unique solution of a p-Laplacian fractional differential equation with singular decreasing nonlinearity. Bound. Value Probl. 2018, Article ID 82 (2018)

37. Zhao, Y., Hou, X., Sun, Y., Bai, Z.: Solvability for some class of multi-order nonlinear fractional systems. Adv. Differ. Equ. 2019, Article ID 23 (2019)

38. Ma, W., Cui, Y.: The eigenvalue problem for Caputo type fractional differential equation with Riemann-Stieltjes integral boundary conditions. J. Funct. Spaces 2018, Article ID 2176809 (2018)

39. Qi, T., Liu, Y., Zou, Y.: Existence result for a class of coupled fractional differential systems with integral boundary value conditions. J. Nonlinear Sci. Appl. 10, 4034-4045 (2017)

40. Qi, T., Liu, Y., Cui, Y.: Existence of solutions for a class of coupled fractional differential systems with nonlocal boundary conditions. J. Funct. Spaces 2017, Article ID 6703860 (2017)

41. Zhang, X., Liu, L., Zou, Y.: Fixed-point theorems for systems of operator equations and their applications to the fractional differential equations. J. Funct. Spaces 2018, Article ID 7469868 (2018) 
42. Zhang, K., Wang, J., Ma, W.: Solutions for integral boundary value problems of nonlinear Hadamard fractional differential equations. J. Funct. Spaces 2018, Article ID 2193234 (2018)

43. Zhai, C., Wang, W., Li, H.: A uniqueness method to a new Hadamard fractional differential system with four-point boundary conditions. J. Inequal. Appl. 2018, Article ID 207 (2018)

44. Zhang, K., Fu, Z.: Solutions for a class of Hadamard fractional boundary value problems with sign-changing nonlinearity. J. Funct. Spaces 2019, Article ID 9046472 (2019)

45. Fu, Z., Bai, S., O'Regan, D., Xu, J.: Nontrivial solutions for an integral boundary value problem involving Riemann-Liouville fractional derivatives. J. Inequal. Appl. 2019, Article ID 104 (2019)

46. Zhang, K., O'Regan, D., Xu, J., Fu, Z:: Nontrivial solutions for a higher order nonlinear fractional boundary value problem involving Riemann-Liouville fractional derivatives. J. Funct. Spaces 2019, Article ID 2381530 (2019)

47. Jiang, J., O'Regan, D., Xu, J., Fu, Z.: Positive solutions for a system of nonlinear Hadamard fractional differential equations involving coupled integral boundary conditions. J. Inequal. Appl. 2019, Article ID 204 (2019)

48. Jiang, J., O'Regan, D., Xu, J., Cui, Y.: Positive solutions for a Hadamard fractional $p$-Laplacian three-point boundary value problem. Mathematics 7(5), 439 (2019)

49. Cheng, W., Xu, J., Cui, Y., Ge, Q.: Positive solutions for a class of fractional difference systems with coupled boundary conditions. Adv. Differ. Equ. 2019, Article ID 249 (2019)

50. Wang, F., Cui, Y., Zhou, H.: Solvability for an infinite system of fractional order boundary value problems. Ann. Funct. Anal. 10(3), 395-411 (2019)

51. Wang, F., Cui, Y.: Unbounded solutions to abstract boundary value problems of fractional differential equations on a half line. Math. Methods Appl. Sci. (2019) in press

52. Wang, F., Cui, Y.: Positive solutions for an infinite system of fractional order boundary value problems. Adv. Differ. Equ. 2019, Article ID 169 (2019)

53. Feckan, M., Zhou, Y., Wang, J.: On the concept and existence of solution for impulsive fractional differential equations. Commun. Nonlinear Sci. Numer. Simul. 17, 3050-3060 (2012)

54. Wang, J., Zhou, Y., Feckan, M.: Nonlinear impulsive problems for fractional differential equations and Ulam stability. Comput. Math. Appl. 64, 3389-3405 (2012)

55. Wang, J., Zada, A., Ali, W.: Ulam's-type stability of first-order impulsive differential equations with variable delay in quasi-Banach spaces. Int. J. Nonlinear Sci. Numer. Simul. 19(5), 553-560 (2018)

56. Zada, A., Ali, W., Farina, S.: Hyers-Ulam stability of nonlinear differential equations with fractional integrable impulses. Math. Methods Appl. Sci. 40(15), 5502-5514 (2017)

57. Wang, Y., Liu, Y., Cui, Y.: Infinitely many solutions for impulsive fractional boundary value problem with $p$-Laplacian. Bound. Value Probl. 2018, Article ID 94 (2018)

58. Zuo, M., Hao, X., Liu, L., Cui, Y.: Existence results for impulsive fractional integro-differential equation of mixed type with constant coefficient and antiperiodic boundary conditions. Bound. Value Probl. 2017, Article ID 161 (2017)

59. Bai, Z., Dong, X., Yin, C.: Existence results for impulsive nonlinear fractional differential equation with mixed boundary conditions. Bound. Value Probl. 2016, Article ID 63 (2016)

60. Riaz, U., Zada, A., Ali, Z., Ahmad, M., Xu, J., Fu, Z.: Analysis of nonlinear coupled systems of impulsive fractional differential equations with Hadamard derivatives. Math. Probl. Eng. 2019, Article ID 5093572 (2019)

61. Riaz, U., Zada, A., Ali, Z., Cui, Y., Xu, J.: Analysis of coupled systems of implicit impulsive fractional differential equations involving Hadamard derivatives. Adv. Differ. Equ. 2019, Article ID 226 (2019)

62. Ulam, S.M.: A Collection of Mathematical Problems. Interscience, New York (1968)

63. Hyers, D.H.: On the stability of the linear functional equation. Proc. Natl. Acad. Sci. USA 27, 222-224 (1941)

64. Rassias, Th.M.: On the stability of linear mappings in Banach spaces. Proc. Am. Math. Soc. 72, 297-300 (1978)

65. Rizwan, R.: Existence theory and stability analysis of fractional Langevin equation. Int. J. Nonlinear Sci. Numer. Simul. https://doi.org/10.1515/ijnsns-2019-0053

66. Shah, R., Zada, A.: A fixed point approach to the stability of a nonlinear Volterra integrodifferential equation with delay. Hacet. J. Math. Stat. 47(3), 615-623 (2018)

67. Shah, S.O., Zada, A., Hamza, A.E.: Stability analysis of the first order non-linear impulsive time varying delay dynamic system on time scales. Qual. Theory Dyn. Syst. https://doi.org/10.1007/s12346-019-00315-x

68. Shah, S.O., Zada, A.: Existence, uniqueness and stability of solution to mixed integral dynamic systems with instantaneous and noninstantaneous impulses on time scales. Appl. Math. Comput. 359, 202-213 (2019)

69. Wang, X., Arif, M., Zada, A.: $\beta$-Hyers-Ulam-Rassias stability of semilinear nonautonomous impulsive system. Symmetry 11(2), 231 (2019)

70. Zada, A., Mashal, A.: Stability analysis of nth order nonlinear impulsive differential equations in quasi-Banach space. Numer. Funct. Anal. Optim. (2019). https://doi.org/10.1080/01630563.2019.1628049

71. Zada, A., Shaleea, S., Li, T.: Stability analysis of higher order nonlinear differential equations in $\beta$-normed spaces. Math. Methods Appl. Sci. 42(4), 1151-1166 (2019)

72. Zada, A., Ali, W., Park, C.: Ulam's type stability of higher order nonlinear delay differential equations via integral inequality of Grönwall-Bellman-Bihari's type. Appl. Math. Comput. 350, 60-65 (2019)

73. Benchohra, M., Graef, J.R., Hamani, S.: Existence results for boundary value problems with nonlinear fractional differential equations. Appl. Anal. 87(7), 851-863 (2008)

74. Ali, Z., Zada, A., Shah, K.: Ulam stability to a toppled systems of nonlinear implicit fractional order boundary value problem. Bound. Value Probl. 2018, Article ID 175 (2018)

75. Wang, J., Zada, A., Waheed, H.: Stability analysis of a coupled system of nonlinear implicit fractional anti-periodic boundary value problem. Math. Methods Appl. Sci. (2019). https://doi.org/10.1002/mma.5773

76. Wang, J., Zhou, Y., Lin, Z: On a new class of impulsive fractional differential equations. Appl. Math. Comput. 242, 649-657 (2014)

77. Zada, A., Ali, S., Li, Y.: Ulam-type stability for a class of implicit fractional differential equations with non-instantaneous integral impulses and boundary condition. Adv. Differ. Equ. 2017, Article ID 317 (2017)

78. Zada, A., Ali, S.: Stability analysis of multi-point boundary value problem for sequential fractional differential equations with non-instantaneous impulses. Int. J. Nonlinear Sci. Numer. Simul. 19(7), 763-774 (2018)

79. Wang, J., Feckan, M., Zhou, Y.: Ulam's type stability of impulsive ordinary differential equation. J. Math. Anal. Appl. 35 258-264 (2012) 
80. Diaz, J.B., Margolis, B:: A fixed point theorem of the alternative, for contractions on a generalized complete matric space. Bull. Am. Math. Soc. 74, 305-309 (1968)

Submit your manuscript to a SpringerOpen ${ }^{\circ}$ journal and benefit from:

- Convenient online submission

- Rigorous peer review

- Open access: articles freely available online

- High visibility within the field

- Retaining the copyright to your article

Submit your next manuscript at $\boldsymbol{~ s p r i n g e r o p e n . c o m ~}$ 